\title{
Imaging in Sarcoidosis
}

\author{
M. Reza Rajebi ${ }^{1}$, Nicole A. Zimmerman ${ }^{1}$, Roozbeh Sharif ${ }^{2}$, \\ Ernest M. Scalzetti ${ }^{1}$, Stuart A. Groskin ${ }^{3}$ and Rolf A. Grage ${ }^{1}$ \\ ${ }^{1}$ SUNY Upstate Medical University, \\ ${ }^{2}$ University of Texas Health Science Center at Houston, \\ ${ }^{3}$ Crouse Hospital, \\ USA
}

\section{Introduction}

Sarcoidosis is an enigmatic systemic granulomatous disease of unknown etiology. It was first described in 1877 by Jonathon Hutchinson, an English physician (Hutchinson, 1877). Caesar Boeck used the term sarkoid originally in 1899 because he felt that the lesions resembled sarcoma (Boeck, 1899). Kuznitsky and Bittorf reported the cardinal patient with enlarged hilar nodes and pulmonary infiltrates on chest radiograph, describing the principle case of sarcoidosis (Kuznitsky \& Bittorf, 1915).

Sarcoidosis primarily affects the lung and lymphatic system. The patients are usually young to middle-aged adults, appearing more commonly in individuals of African descent. Bilateral hilar lymphadenopathy, pulmonary involvement, and ocular and cutaneous lesions are classic findings, however sarcoidosis can affect many other organs. Clinical and imaging findings are helpful in establishing the diagnosis. Histologic demonstration of noncaseating epithelioid cell granulomas is confirmatory for diagnosis. In 1958, Wurm and colleagues proposed a radiographic staging system, which still remains in widespread clinical use (Table 1) (Wurm et al., 1958).

\begin{tabular}{|c|c|}
\hline Stage & Radiographic findings \\
\hline 0 & Normal \\
\hline 1 & Bilateral hilar adenopathy \\
\hline 2 & Bilateral hilar adenopathy with parenchymal opacities \\
\hline 3 & Parenchymal opacities \\
\hline 4 & Advanced lung disease with fibrosis \\
\hline
\end{tabular}

Table 1. Radiographic stages of pulmonary sarcoidosis as defined by chest radiograph.

This chapter reviews the imaging findings of sarcoidosis based on the involved organ systems and recommends the principle imaging study contingent upon the diagnostic capabilities of different modalities. Options for radiologic evaluation of sarcoidosis include plain radiography, ultrasonography, scintigraphy, computed tomography (CT), and magnetic resonance imaging (MRI). Until the 1970s, evaluation of sarcoidosis was limited to plain chest radiographs. Radionuclide imaging is occasionally used to assess lymphatic, cardiac, and musculoskeletal involvement. With the development of CT in the 
early 1970s, cross-sectional imaging became available. The multiplanar and multisequential capabilities of MRI have enhanced the characterization of sarcoid lesions involving different organs.

\section{Thoracic sarcoidosis}

Classically, thoracic involvement of sarcoidosis presents as bilateral hilar adenopathy which may occur in combination with parenchymal opacities. The chest roentgenogram is still the most commonly used method for detecting lung involvement, however various other modalities are being used with increased frequency. Pulmonary involvement is present in $90 \%$ of patients with sarcoidosis.

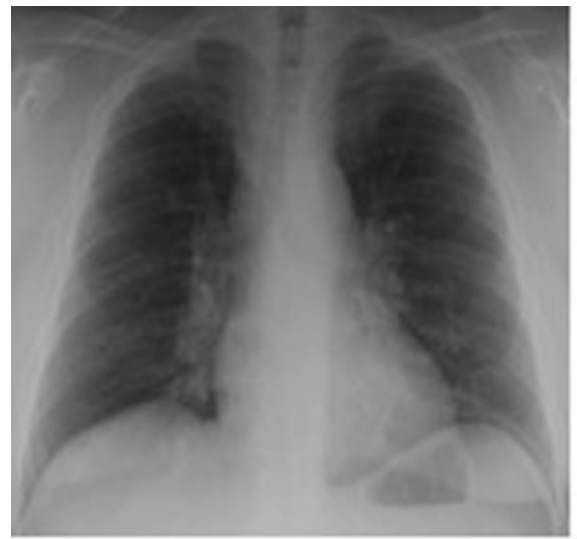

(a)

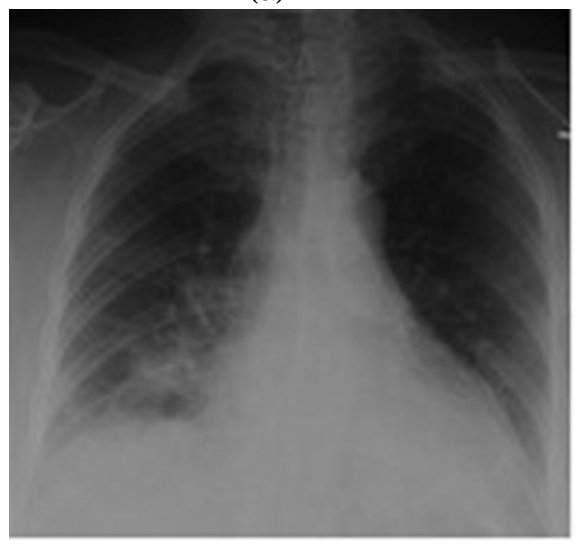

(c)

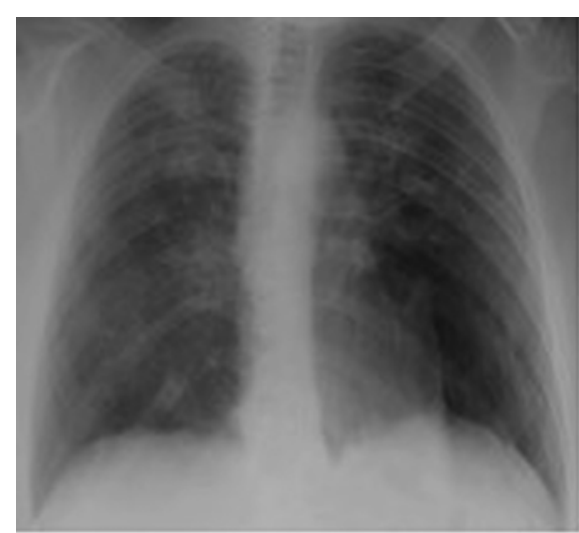

(b)

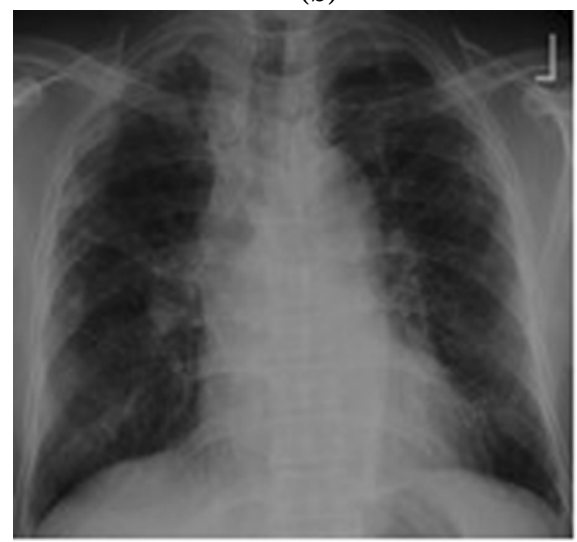

(d)

Fig. 1. Radiographic stages of pulmonary sarcoidosis. (a) Stage 1: A 32-year-old female with hilar and right paratracheal lymphadenopathy. (b) Stage 2: A 61-year-old male with bilateral hilar adenopathy and parenchymal opacities. (c) Stage 3: A 54-year-old female with parenchymal opacities in the right lower lung. (d) Stage 4: A 51-year-old female with extensive increased interstitial markings. 


\subsection{Radiography}

Simple radiographs are sufficient for diagnosing and follow-up of sarcoidosis, although highresolution CT without intravenous contrast is helpful in characterizing interstitial lung disease and the extent of adenopathy. It is also suitable in determining the lung involvement stage, amount and type of reversible versus irreversible lung damage, and the overall prognosis.

Pulmonary involvement can be categorized into five radiographic stages (Figure 1) (Silzbach, 1967). Pulmonary function test findings such as restrictive pattern and decreased carbon monoxide diffusing capacity worsen as the disease progresses from stages zero to four (Criado et al., 2010).

Lymphadenopathy is the most common finding in patients with pulmonary sarcoidosis; it is seen in $80 \%$ of patients. Bilateral hilar lymphadenopathy is the typical pattern of involvement. Additionally, right paratracheal lymphadenopathy is also common. Lymph nodes can be of different sizes ranging from subtle enlargement to conglomerate masses.

In $20 \%$ of patients, parenchymal infiltration can be identified on radiographs. Reticulonodular opacities are the most common findings and are primarily seen in the upper lung zones. Large nodules are seen in patients with alveolar sarcoidosis. In cases with advanced disease and fibrosis, honeycombing and traction bronchiectasis may be seen. The upper lobes and superior segments of the lower lobes are usually involved in this presentation. Occasionally, cavitary lesions and pleural effusion can be demonstrated.

\subsection{Computed tomography}

High-resolution CT is more sensitive in detecting adenopathy and parenchymal involvement as well as differentiating reversible inflammatory features from irreversible fibrotic changes. The most common CT findings of pulmonary sarcoidosis are symmetric lymphadenopathy, micronodules with lymphagitic spread, fibrotic changes, and bilateral perihilar opacities. Reversible inflammatory changes consist of nodules and uncommonly, alveolar densities and patchy ground-glass opacities. Irreversible changes due to pulmonary fibrosis include honeycombing, architectural distortion, bullae formation and tractional bronchiectasis.

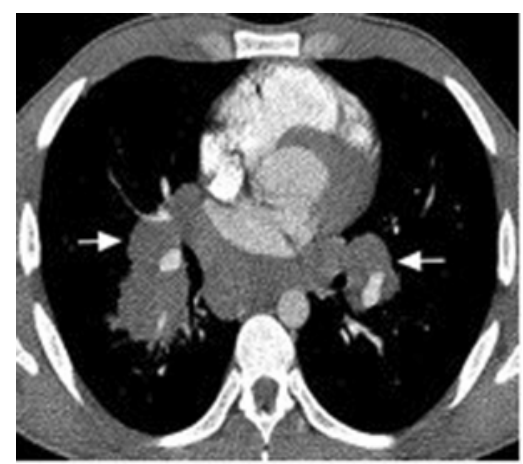

Fig. 2. A 56-year-old female with hilar lymphadenopathy.

Typically, hilar and right paratracheal lymph nodes are enlarged (Figure 2). Furthermore, as the disease progresses to a chronic state, nodes can become calcified (Figure 3). A symmetric bilateral perilymphatic micronodular distribution, commonly referred to as lymphangitic spread, is the most common pattern in patients with parenchymal involvement in pulmonary sarcoidosis (Lynch et al., 1997). Lymphangitic spread can include peribronchovascular, subpleural, and interlobular septal distribution of the nodules (Figure 
4). Micronodules are defined as round nodules measuring 2-4 $\mathrm{mm}$ and usually seen in the upper and middle zones. Bilateral perihilar opacities are one of the common findings in thoracic sarcoidosis; these opacities are confluent nodular consolidations with irregular borders and central predilection (Figure 5).

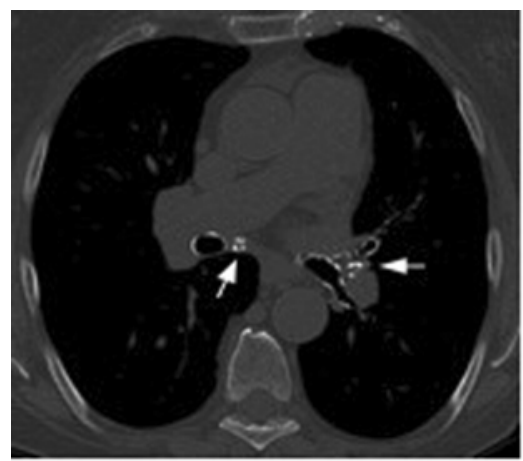

Fig. 3. A 69-year-old female with calcified hilar lymph nodes.

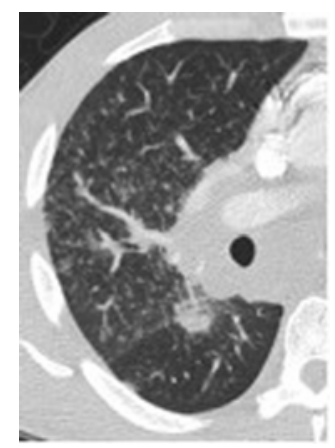

Fig. 4. A 56-year-old female with pulmonary sarcoidosis. CT of the chest demonstrates micronodules with peribronchovascular distribution.

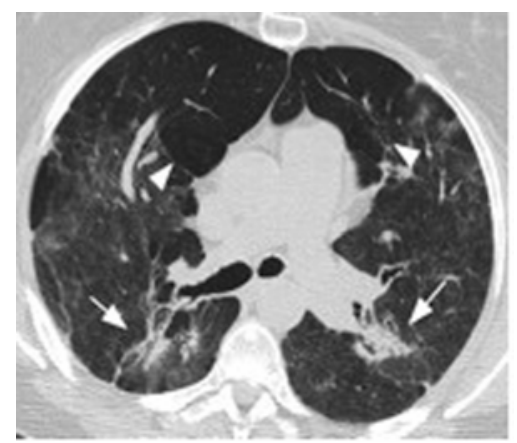

Fig. 5. A 51-year-old female with sarcoidosis. CT of the chest shows bilateral perihilar densities (arrows). Areas of air trapping and mosaic attenuation pattern are also identified (arrowheads) 
In $20 \%$ of patients, the initial inflammatory phase leads to fibrotic changes such as honeycombing, linear densities, architectural distortion and secondary traction bronchieactasis (Figure 6). Honeycombing refers to "destroyed and fibrotic lung tissue containing numerous cystic airspaces with thick fibrous walls, representing the late stage of various lung diseases, with complete loss of acinar architecture" (Hansell et al., 2008). Upper and middle zones are usually involved in a patchy distribution. Abehsera et al described three major patterns of pulmonary involvement in patients with fibrosis: bronchial distortion in $47 \%$ of patients; honeycombing in $29 \%$ of patients; and diffuse linear opacities in $24 \%$ of patients (Abehsera et al., 2000).

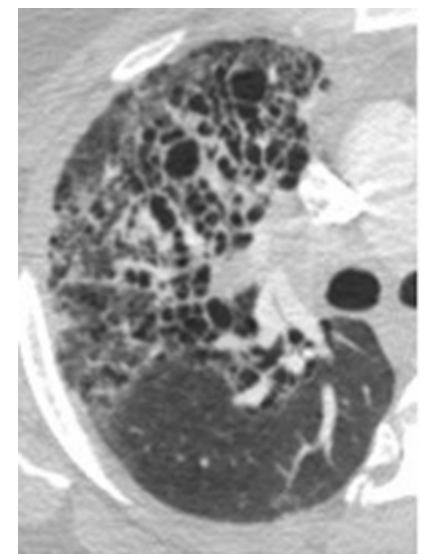

(a)

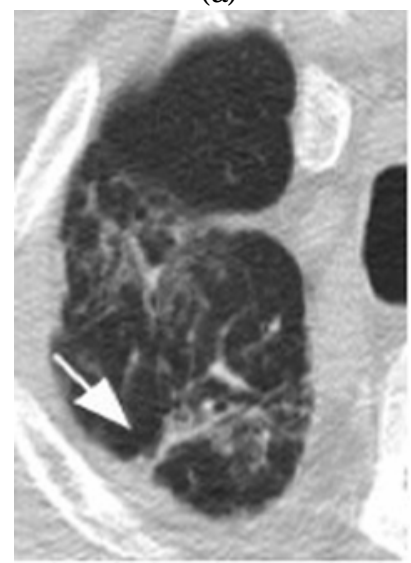

(c)

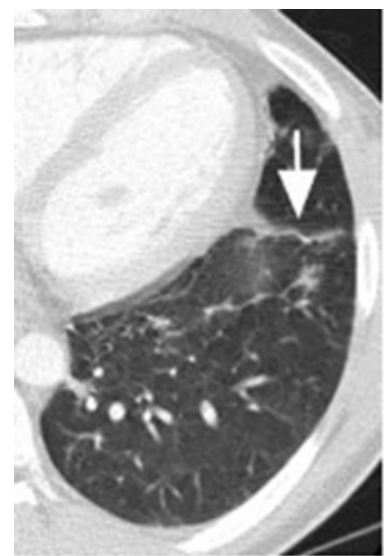

(b)

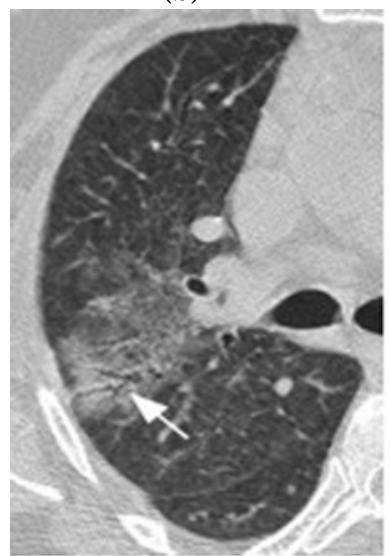

(d)

Fig. 6. Fibrotic changes in advanced sarcoidosis. (a) A 29-year-old female with sarcodidosis. $\mathrm{CT}$ of the chest demonstrates multiple cystic lesions within the left upper lobe, representing areas of honeycombing. (b) A 34-year-old male with sarcoidosis. CT of the chest shows areas of linear densities (arrow) in the left low er lobe compatible with linear scarring. (c) A 49year-old male with sarcoidosis. CT of the chest demonstrates architectural distortion in the right lung apex (arrow). (d) A 54 year-old female with sarcoidosis. CT of the chest shows bronchiectasis in the right upper lobe. 
Atypical features of pulmonary involvement include unilateral lymphadenopathy, unusual location of lymphadenopathy, nodules and masses, patchy airspace, ground-glass, and linear opacities, airway involvement, fibrocystic changes, miliary opacities, and pleural disease. Unusual manifestations of mediastinal lymphadenopathy in a minority of patients may contribute to challenging diagnostic dilemmas.

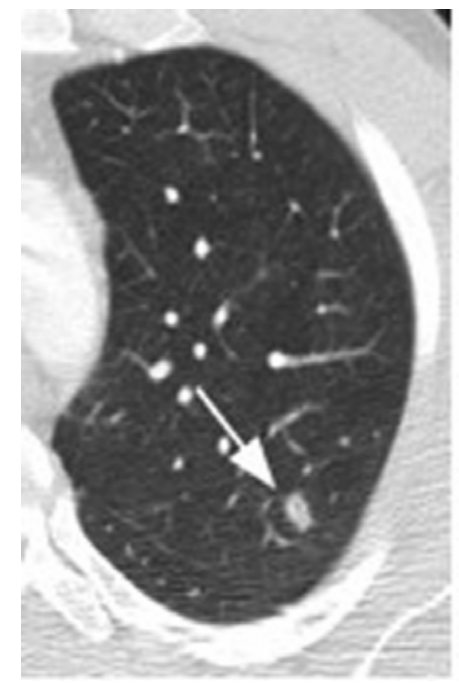

(a)

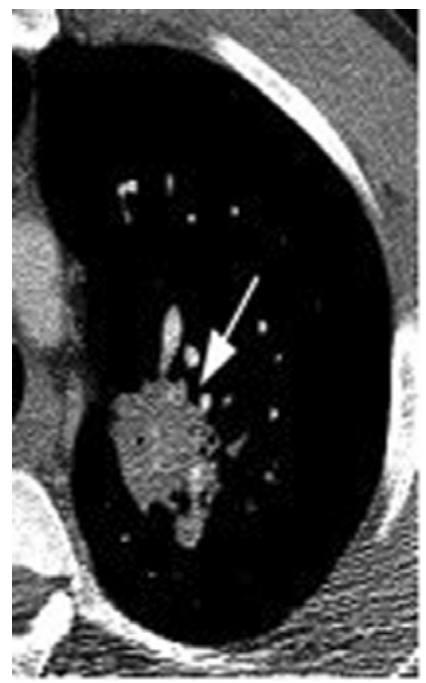

(b)

Fig. 7. Pulmonary macronodules and masses in sarcoidosis. (a) A 34-year-old male. CT of the chest shows a $12 \mathrm{~mm}$ nodule in the left upper lobe. (b) A 39-year-old male. CT of the chest demonstrates a large soft tissue mass in the left upper lobe. Biopsy confirmed noncaseating granulomatous disease in both patients.

Nodules and masses are predominantly bilateral and multiple (Figure 7). Nevertheless, these lesions can occasionally present in varying manners. "Galaxy sign" refers to multiple small nodules around a larger mass (Figure 8). A conglomerate of multiple micronodules is called a "sarcoid cluster". Acinar or alveolar sarcoidosis refers to alveolar opacities, which are composed of multiple nodules causing adjacent alveoli compression and can easily be mistaken as pneumonia, tuberculosis, or cryptogenic organizing pneumonitis (Figure 9). Patchy ground-glass opacities are more commonly seen as compared to diffuse groundglass opacities (Figure 10). Interlobular and intralobular septal thickening may lead to a linear reticular pattern (Figure 11). Honeycomb-like cysts, cavitations and mycetoma formations are considered fibrocystic changes.

Airway involvement is indicative of a poor prognosis (Handa et al., 2006). It can present as mosaic attenuation pattern, air trapping (Figure 5), tracheobronchial abnormalities, and atelectasis. Miliary pattern is a rare presentation of pulmonary sarcoidosis, found in less than one percent of cases (Figure 12). Pleural effusion, hemorrhagic or chylous pleural effusion, pneumothorax (Figure 12), pleural thickening, plaque-like opacities and pleural calcification occur in 1-4\% of patients (Soskel \& Sharma, 1992). 


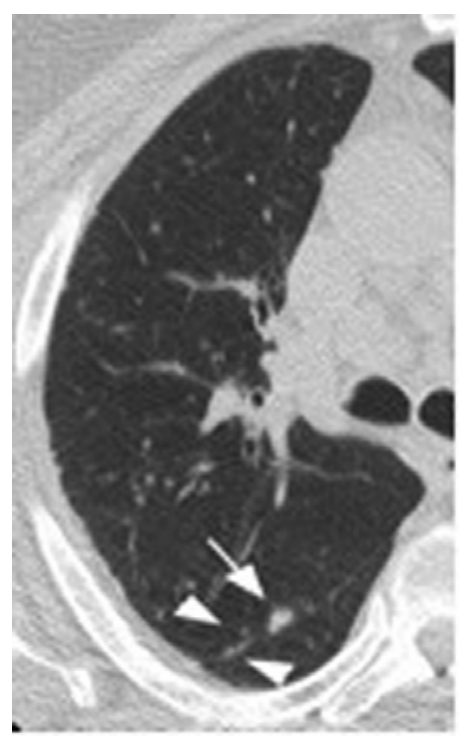

Fig. 8. "Galaxy sign”. A 49-year-old female. CT of the chest shows a $7 \mathrm{~mm}$ nodule in the left lower lobe (arrow) as well as multiple adjacent $2 \mathrm{~mm}$ nodules (arrowhead).

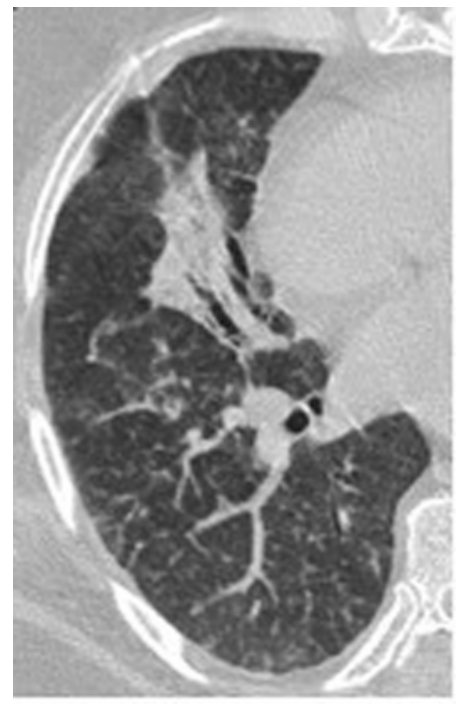

Fig. 9. A 54-year-old female with sarcoidosis. CT of the chest shows a large alveolar density in the right middle lobe 


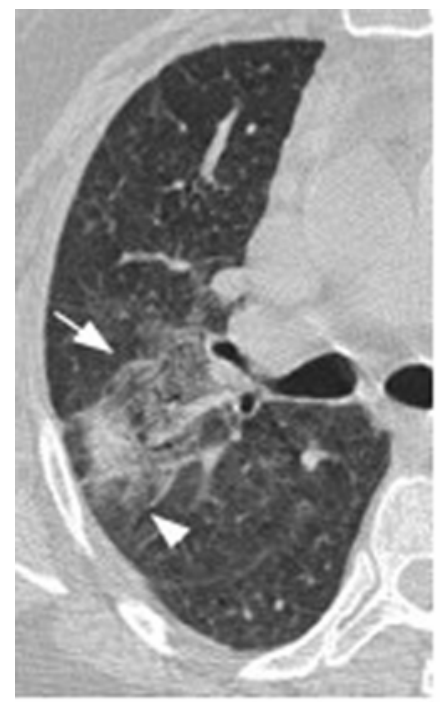

Fig. 10. A 54-year-old female with sarcoidosis. CT of the chest shows an area of patchy groundglass opacity (arrow) in the right upper lobe. A confluent opacity (arrowhead) lateral to this area is also identified.

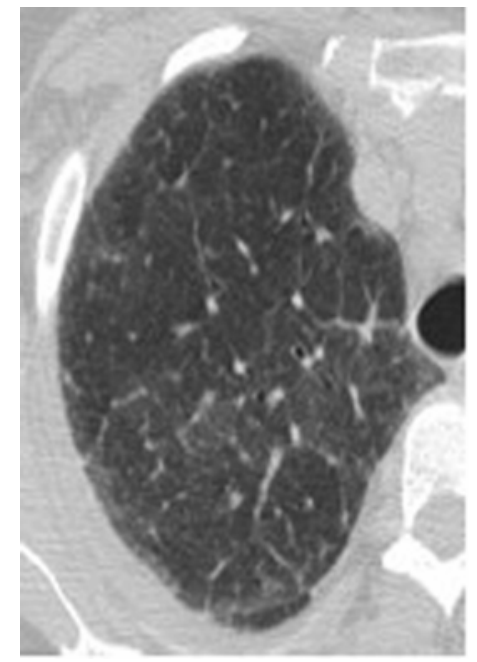

Fig. 11. A 56-year-old female with sarcoidosis. CT of the chest demonstrates intralobular septal thickening in the right upper lobe. 


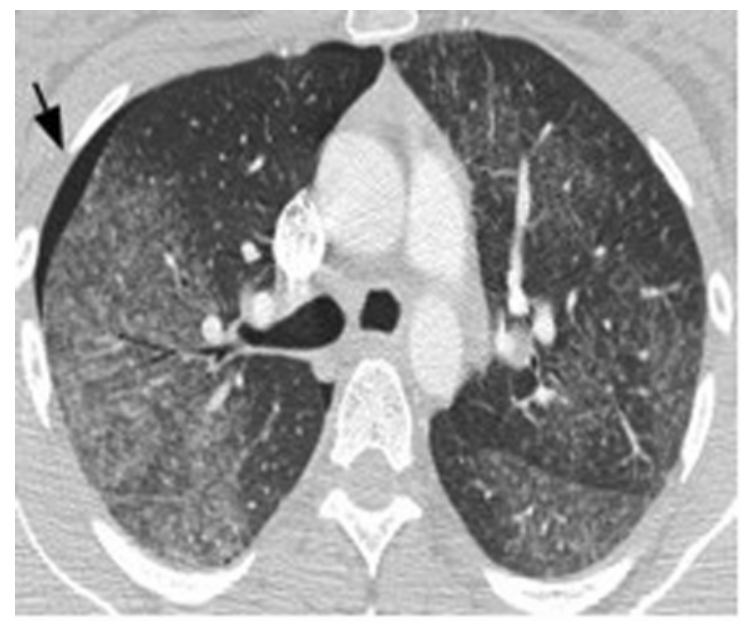

Fig. 12. A 66-year-old male. CT of chest reveals innumerable micronodules with a miliary pattern. A small right-sided pneumothorax (arrow) is also noted.

One study compared the high-resolution CT and pulmonary function test findings of smokers and nonsmokers with pulmonary sarcoidosis. 46 patients ( 23 smokers and 23 lifelong nonsmokers) with histologically proven sarcoidosis were included. Air trapping and small nodules were the most common findings. The extent of emphysema was greater in smokers although no significant difference was seen in the extent of airway involvement between smokers and nonsmokers (Terasaki et al., 2005).

\subsection{Nuclear medicine}

Gallium-67 $\left.{ }^{67} \mathrm{Ga}\right)$ scintigraphy shows a relatively specific pattern of abnormal radiopharmaceutical uptake in thoracic lymph nodes of patients with pulmonary sarcoidosis referred to as "lambda sign". This is a distinctive pattern of increased ${ }^{67} \mathrm{Ga}$ uptake in the hilar and right paratracheal lymph nodes resembling the Greek letter lambda $(\lambda)$. Sulivak et al evaluated 65 patients with sarcoidosis and compared ${ }^{67} \mathrm{Ga}$ scintigraphy findings in these patients with 540 patients with other disorders. Lambda sign was seen in $72 \%$ of patients with sarcoidosis and none of the patients in the comparison group (Sulavik et al., 1990).

\subsection{Ultrasonography}

Endobronchial ultrasound (EBUS) with transbronchial needle aspiration (TBNA) is a promising minimally invasive technique, which recently has been used for diagnosis of a wide variety of pulmonary disorders ranging from sarcoidosis to lung cancer. In one study, EBUS-TBNA is performed for diagnosis of sarcoidosis in 50 patients with suspected pulmonary sarcoidosis. Non-caseating granuloma was demonstrated in 41 of 48 patients (85\%) with a final diagnosis of sarcoidosis (Garwood et al., 2007).

In summary, plain radiographs are sufficient for diagnosis of pulmonary sarcoidosis and subsequent follow-up. High resolution CT without intravenous contrast may be helpful to characterize interstitial lung disease and the extent of adenopathy. 


\section{Cardiac sarcoidosis}

Although clinical cardiac involvement in sarcoidosis is uncommon, it is still a major contributing factor in disease prognosis (Perry \& Vuitch, 1995). Acute cardiac failure, ventricular arrhythmias, heart blockage, and sudden cardiac death are several complications of sarcoidosis in the cardiovascular system. Myocardial involvement is more common than pericardial disease. The basal and lateral walls of the left ventricle as well as the septum are typically involved, while the endocardium is usually spared. As with any other organ, cardiac sarcoidosis can be divided into three stages including initial edema, granulomatous infiltration and scarring.

\subsection{Nuclear medicine}

Resting Thallium-201 and Tc-99m-methoxyisobutylisonitrile (Tc-99m-MIBI) scintigraphy demonstrate segmental regions of decreased radiopharmaceutical uptake in the myocardium although these foci do not correspond to histologic findings for an unknown reason. Reverse distribution has been described in patients with cardiac sarcoidosis as indicated by increased uptake in Thallium-201 on stress images.

${ }^{67} \mathrm{Ga}$ scintigraphy is useful for diagnosis of the active inflammatory phases of cardiac sarcoidosis including the initial edema and granulomatous infiltration phases. Presence of areas of increased uptake in ${ }^{67} \mathrm{Ga}$ scintigraphy correlates with a favorable response to corticosteroid treatment.

18F-fluorodeoxyglucose (FDG) accumulates in inflammatory cells and although it is nonspecific, FDG-Positron Emission Tomography (PET) may be a useful modality for detecting inflammatory processes such as sarcoidosis. Okumura et al (Okumura et al., 2004) described that fasting FDG-PET can detect cardiac sarcoidosis in earlier stages in comparison to other radiopharmaceuticals.

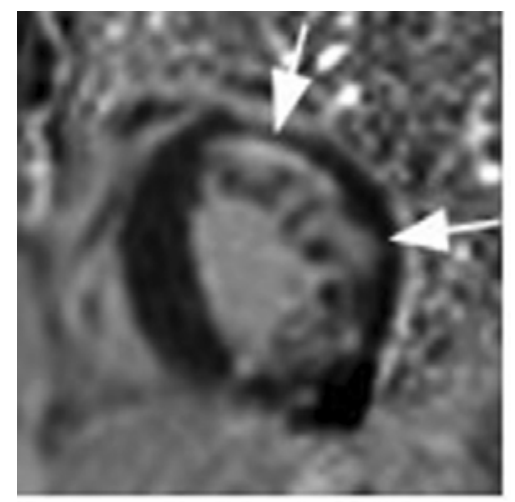

Fig. 13. Short-axis gadolinium-enhanced T1-weighted image shows an area of diffuse enhancement (arrows) in the lateral wall of the left ventricle representing edema in the early stage of cardiac sarcoidosis. (Courtesy of Prof. Olivier Vignaux, University René Descartes, Paris, France) 


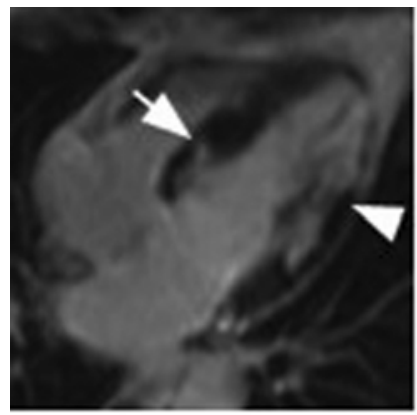

Fig. 14. Four-chamber delayed gadolinium-enhanced T1-weighted image demonstrates an area of nodular enhancement (arrow) in the septum compatible with granulomatous infiltration. Diffuse lateral wall enhancement (arrowhead) is also noted. (Courtesy of Prof. Olivier Vignaux, University René Descartes, Paris, France)

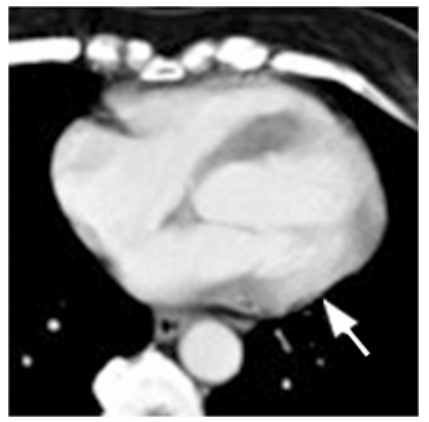

Fig. 15. Axial CT image of the chest in a patient with sarcoidosis shows an area of diffuse enhancement in the lateral wall of the left ventricle, representing presumed cardiac involvement. (Courtesy of Dr. Takashi Koyama, Kyoto University of Medicine, Kyoto, Japan)

\subsection{Magnetic resonance imaging}

Magnetic resonance imaging is a sensitive method for diagnosis of cardiac sarcoidosis. Initial edema will present as regions of increased signal intensity on T2-weighted sequences and early contrast enhancement within the myocardium (Figure 13). Granulomatous infiltration can be visualized as nodular areas of signal intensity on T2-weighted images with delayed contrast enhancement (Figure 14). Eventually focal areas of wall thinning and hypokinesis may appear representing a progression into the third phase, scarring. These lesions correspond to foci of delayed enhancement in the subepicardial layer. Segmental contraction abnormalities can be seen, however severe disease can lead to diffuse contraction abnormalities and wall thickening.

Ichinose et al performed delayed contrast-enhanced cardiac MRI in 40 patients with sarcoidosis, 11 having cardiac involvement (Ichinose et al., 2008). Myocardial enhancement was present in $91 \%$ of patients with cardiac sarcoidosis (10 of the 11), whereas none of the 29 patients without cardiac sarcoidosis showed enhancement. They concluded that myocardial lesions can be predominantly localized in the basal and subepicardial myocardium. 
Clinically, cardiac sarcoidosis is found in five percent of patients, however it is present in approximately $20-30 \%$ of autopsy studies indicating that often it can be asymptomatic. In those patients that display clinical symptoms, cardiac sarcoidosis can be life-threatening and detection by MRI becomes increasingly important. MRI with gadolinium is the forefront modality in following cardiac sarcoidosis (Vignaux, 2005). CT is not a commonly used modality for evaluation of cardiac sarcoidosis. Nevertheless, in a few case reports, CT reveals a similar pattern of myocardial enhancement as in MRI (Figure 15).

\section{Abdominal sarcoidosis}

Abdominal visceral involvement is frequently reported in autopsy series of patients with sarcoidosis (Iwai et al., 1988). Nonetheless, most of these patients are asymptomatic and organ dysfunction is uncommon. Abdominal lymphadenopathy as well as genitourinary, pancreatic, and gastrointestinal sarcoidosis have been documented with the liver and spleen being the most frequently affected abdominal organs.

\subsection{Liver and spleen}

Hepatomegaly is the most common imaging finding of hepatic sarcoidosis. Liver dysfunction is occasionally seen; how ever end stage liver disease manifesting as cirrhosis or portal hypertension is rarely reported. On ultrasonography, hepatic sarcoidosis could represent as diffuse or patchy areas of increased echogenicity although the enlarged liver might remain homogenous. Another frequently seen pattern of involvement presents as multiple low-density lesions throughout the liver. These lesions can range from 1-2 millimeters to several centimeters. Sarcoidosis nodules could present as hypoechoic or hyperechoic lesions on sonographic examination. These nodules remain unenhanced on contrast-enhanced CT (Figure 16) and present as low signal intensity nodules with no evidence of enhancement on MRI. Rarely, cirrhosis can be seen with involvement of intrahepatic biliary ducts. When involvement includes the extrahepatic ducts, granulomatous infiltration can lead to obstructive jaundice.

Splenomegaly is a common finding of sarcoidosis, reported in up to $33 \%$ of patients (Warshauer \& Lee, 2004). Hypodense nodular disease is frequently seen while calcified lesions have also been reported (Figure 17). Nodular splenic disease has similar imaging characteristic as hepatic nodular disease (Figure 18).

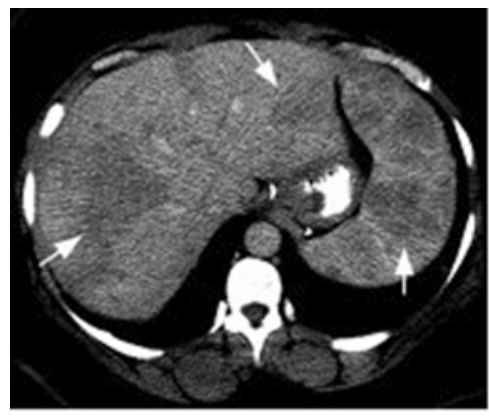

Fig. 16. A 41-year-old female with upper abdominal pain. Axial contrast-enhanced CT image of the abdomen at the level of the T11 vertebra reveals low attenuating geographic lesions (arrows) in the liver and spleen. Splenomegaly is also present. Biopsy confirmed noncaseating granulomas. 


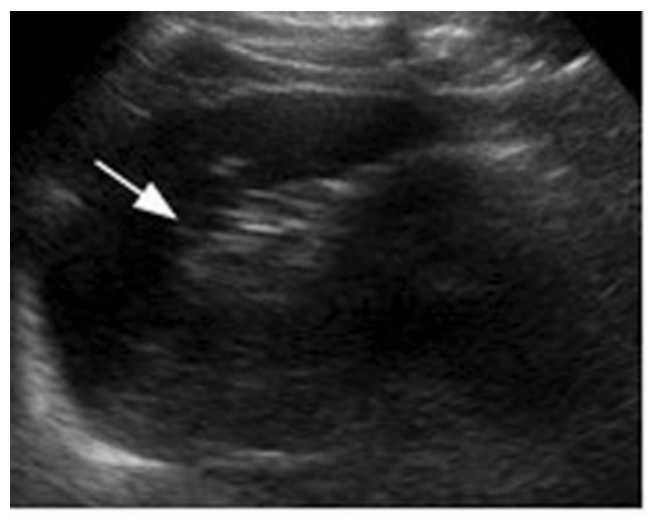

Fig. 17. A 47-year-old female with systemic sarcoidosis. Ultrasonography image of the spleen shows a hyperechoic lesion withon the parenchyma compatible with a calcified splenic granuloma.

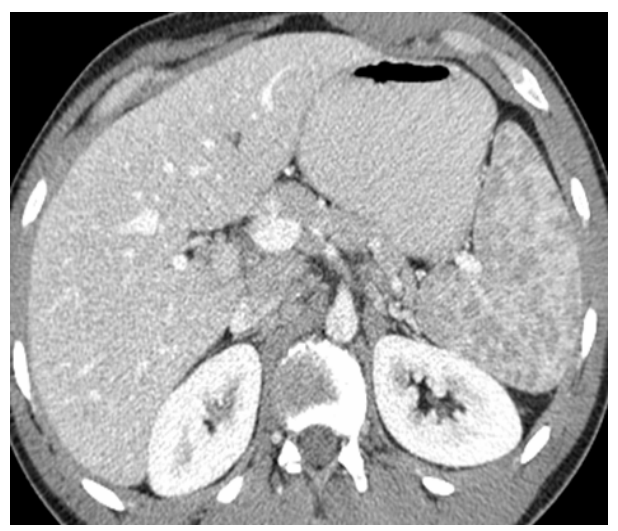

Fig. 18. A 30-year-old male with systemic sarcoidosis. Axial contrast-enhanced CT image shows multiple small low attenuating nodules throughout the spleen.

\subsection{Lymph nodes}

Lymphadenopathy is another common finding of abdominal sarcoidosis. Constitutional symptoms such as fever, fatigue and weight loss have been reported. In comparison with lymphoma, one study showed smaller nodes and less conglomerate mass formation in abdominal lymphadenopathy of sarcoidosis (Britt et al., 1991). Enlarged lymph nodes can compress on adjacent organs causing obstruction of hollow organs such as the biliary ducts or ureters leading to obstructive jaundice or hydronephrosis. These nodes appear hypoechoic on ultrasonography (Figure 19a), of soft tissue density on CT (Figure 19b), and mildly enhancing with increased T2 signal intensity on MRI. 


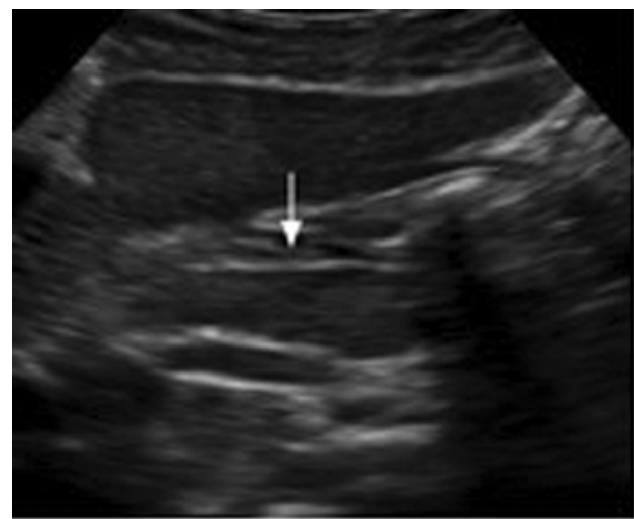

(a)

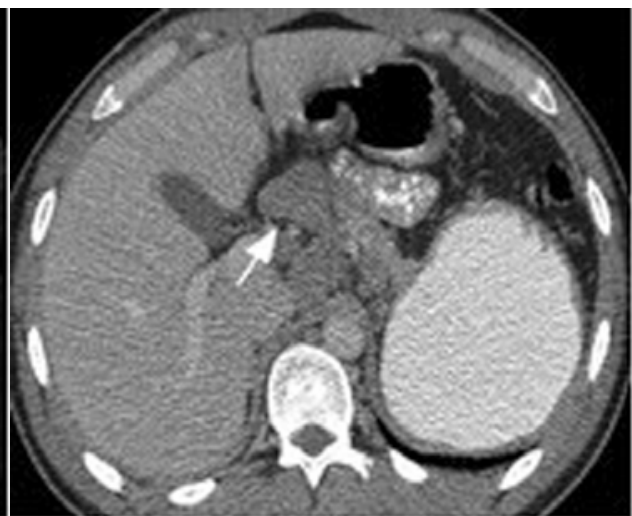

(b)

Fig. 19. Abdominal lymphadenopathy. (a) A 34-year-old male with jaundice and systemic sarcoidosis. An ultrasonography image of the midline upper abdomen shows an enlarged lymph node (arrow) near the porta hepatis. (b) Axial contrast-enhanced CT image of the upper abdomen confirms intrabdominal lymphadenopathy (arrow).

\subsection{Pancreas}

Pancreatitis due to granulomatous infiltration of the pancreatic duct secondary to sarcoidosis is an uncommon manifestation of sarcoidosis. Additionally, focal pancreatic masses have been reported. These masses appear hypoechoic on ultrasonography and hypodense on CT. These lesions typically do not enhance or enhance minimally on CT.

\subsection{Gastrointestinal tract}

Sarcoidosis can involve any part of the gastrointestinal tract. In the stomach focal nodular involvement with wall thickening is a classic finding. Gastric wall ulceration is another manifestation of sarcoidosis. Duodenal, colonic and appendiceal involvement have also been reported. Focal wall thickening is the most common imaging presentation.

\subsection{Genitourinary tract}

Hypercalcemia and hypercalciuria in the setting of sarcoidosis can lead to nephrocalcinosis, nephrolithiasis, and interstitial calcium deposition, which can be visualized on CT and ultrasonography. On CT, multiple isodense lesions with relative hypo-enhancement compared to normal renal parenchyma has been reported. Testicular involvement is rare, appearing as hypodense lesions on CT and hypoechoic areas on ultrasonography (Figure 20).

Organomegaly and nodular disease are the common manifestations of abdominal sarcoidosis. How ever, these findings are generally nonspecific and could be present in other settings such as neoplasms and infectious diseases. A combination of imaging findings and clinical signs and symptoms will help with further delineation of the disease process and possible need for tissue biopsy. 


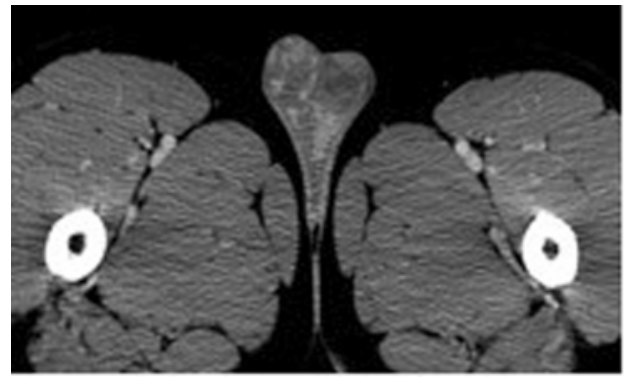

(a)

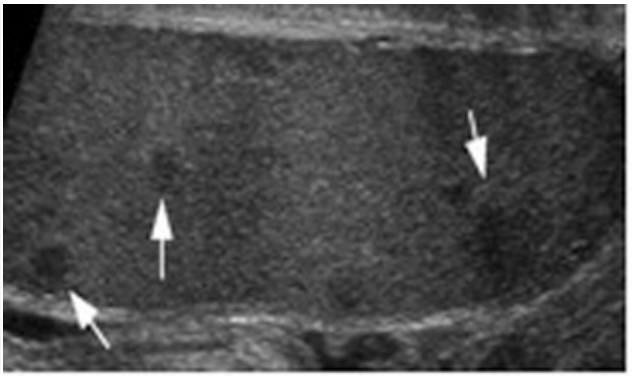

(b)

Fig. 20. A 30-year-old male with sarcoidosis. (a) Axial contrast-enhanced CT image incidentally shows hypodense lesions within the testes. (b) Subsequent sagittal ultrasonography image of the testes show multiple small round hypoechoic lesions (arrow).

\section{Neurosarcoidosis}

Symptomatic involvement of the nervous system is reported in 5\% of patients with sarcoidosis. However, asymptomatic neurosarcoidosis has been reported in up to $25 \%$ of cases in postmortem studies (Jhhns \& Michele, 1999). Isolated neurosarcoidosis is rarely seen. Although sarcoidosis can involve the entire nervous system and its supportive tissues, Central Nervous System (CNS) involvement is far more common. Clinical symptoms of neurosarcoidosis are nonspecific; the involved region of the CNS determines the presenting symptoms. Vision loss, facial nerve involvement, headache, neck stiffness and seizure have all been described as correlated symptoms. Imaging characteristics of neurosarcoidosis are nonspecific so a lesion biopsy is occasionally performed to reach a definite diagnosis.

\subsection{Leptomeningeal involvement}

Enhancing thickened leptomeninges on contrast-enhanced T1-weighted sequences are the most common imaging finding of CNS involvement with sarcoidosis; it has been reported in $40 \%$ of patients with neural involvement (Nowak \& Widenka, 2001). Nodular and diffuse patterns have been described with the basilar meninges being most commonly involved (Figure 21). Some authors have hypothesized that parenchymal involvement is an extension of leptomeningeal disease via the perivascular spaces (Junger et al., 1993). In the spinal cord linear and nodular enhancing extramedullary lesions are also common. Hydrocephalus secondary to adhesions or abnormal CSF resorption can be seen in patients with leptomeningeal sarcoidosis.

\subsection{Parenchymal disease}

Multiple high signal intensity lesions on T2-weighted sequences are the most common finding in patients with parenchymal involvement. In the brain, these lesions are mainly located in the periventricular white matter. Although non-enhancing lesions are typical, contrast enhancement can also be seen (Figure 22). Occasionally, low signal intensity masses on T2-weighted sequences have been reported. 


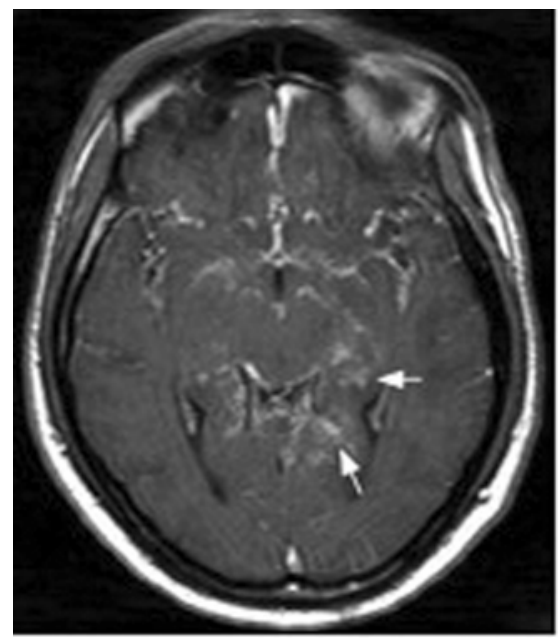

(a)

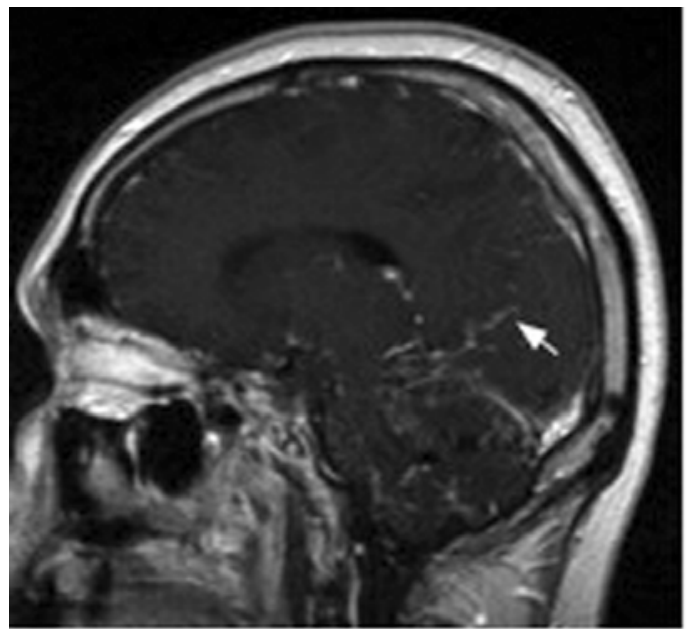

(b)

Fig. 21. Leptomeningeal sarcoidosis. (a) A gadolinium-enhanced axial T1-w eighted image in a 27-year-old male and (b) a gadolinium-enhanced sagittal T1-weighted image in a 38-yearold male with systemic sarcoidosis show curvilinear areas of abnormal enhancement along the sulci and basal cisterns, representing leptomeningeal sarcoidosis.

In the spinal cord, intramedullary sarcoidosis presents as fusiform high T2-signal intensity segmental lesions with decreased signal intensity on T1-weighted sequences. Patchy enhancement on contrast-enhanced T1-weighted sequences is common (Figure 23). One study evaluated clinical, laboratory, and MRI profiles in 31 patients with spinal cord sarcoidosis (Cohen-Aubart et al., 2010). Spinal cord MRIs performed in 26 patients with spinal cord involvement revealed intramedullary T2-hyperintensities that were extensive and heterogeneous with a central distribution in axial slides. They concluded that a spinal cord MRI study may be a useful tool in the diagnosis of spinal cord sarcoidosis.

Cranial nerve involvement is another feature of parenchymal sarcoidosis with contrast enhancing nerve enlargement being typical. Optic nerve involvement is frequently seen and can be bilateral or unilateral (Figure 24). In MRI scans of 15 patients with orbital or optic pathway involvement, eight patients had MR evidence of optic nerve involvement by sarcoid granuloma (Carmody et al., 1994). Optic chiasmal involvement, periventricular white matter abnormalities, perineural enhancement, and optic nerve enlargement were commonly identified. Less frequently, optic nerve atrophy, increased T2 signal intensity in the optic radiations, and orbital masses with MR signal characteristics similar to a pseudotumor were reported.

Additionally, other orbital structures such as orbital fat, muscles, globe and lacrimal glands can be involved (Figure 25). Mass-like lacrimal and muscle infiltration, pseudotumor-like intraorbital masses and rarely uveal and scleral nodularity have been reported. Facial nerve paralysis can be seen with facial nerve (VII) involvement. Nevertheless, there is no correlation between imaging findings and clinical presentation. 


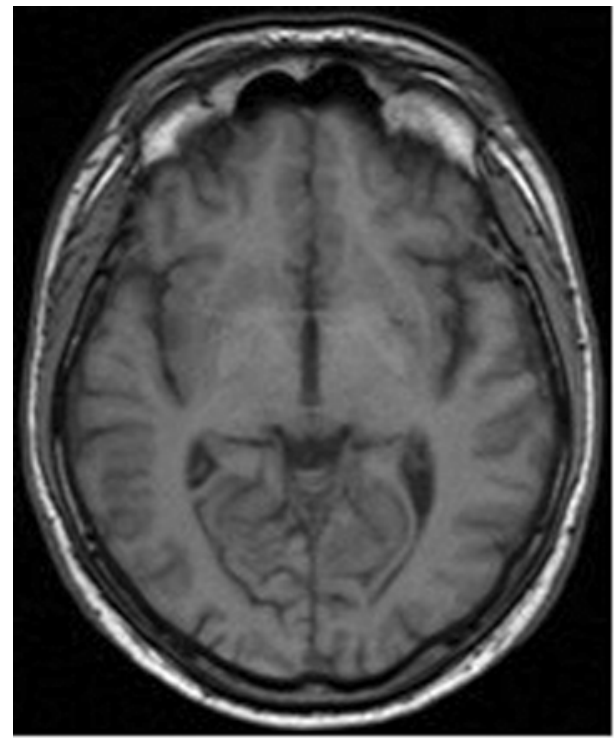

(a)

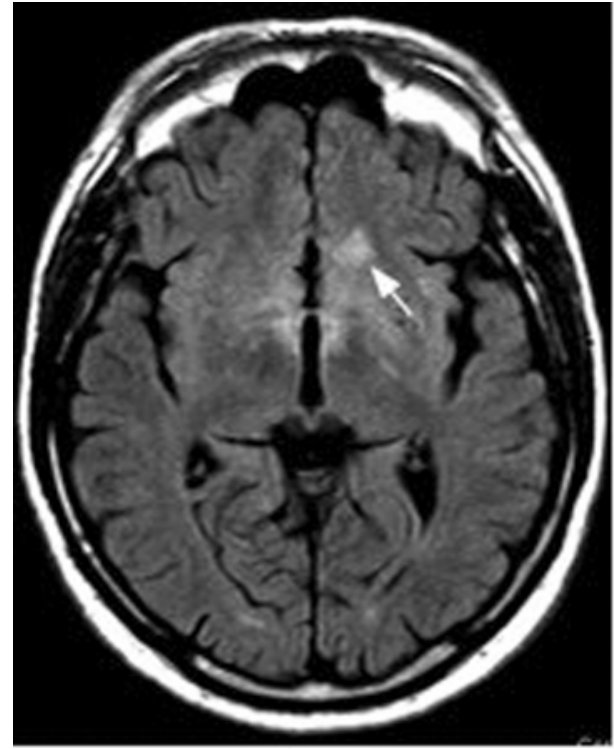

(c)

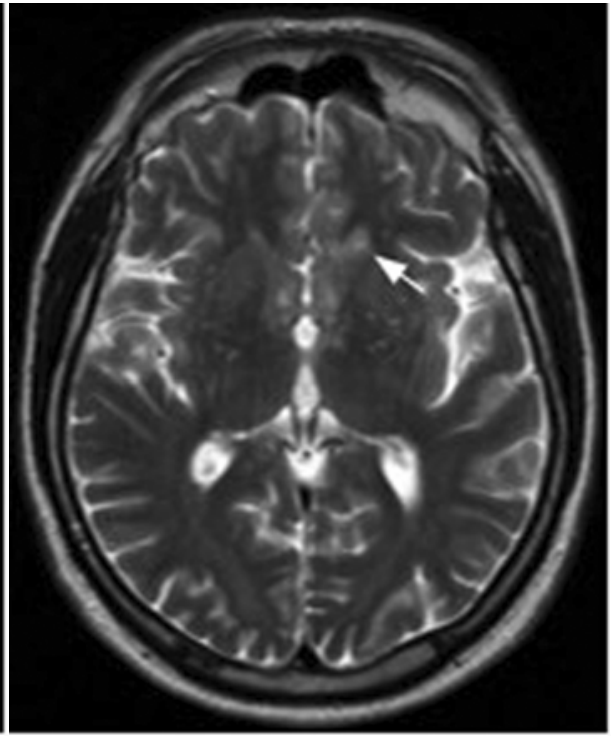

(b)

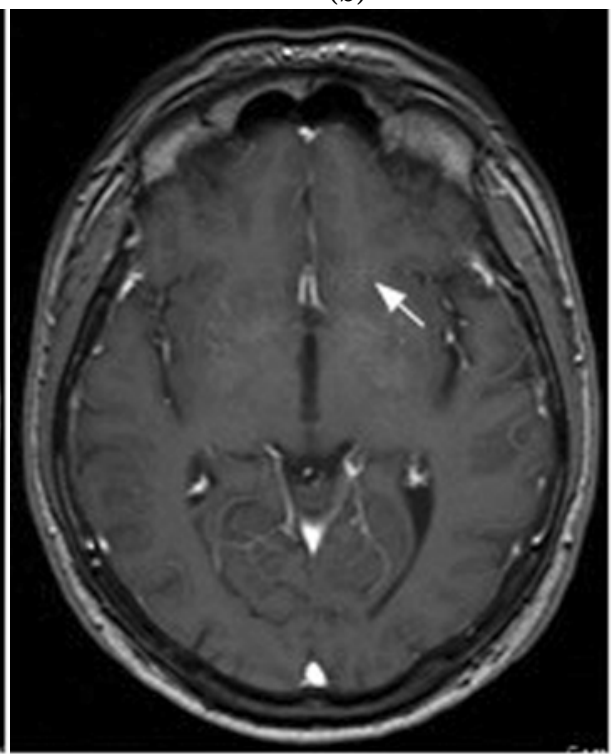

(d)

Fig. 22. A 40-year-old male with memory loss, confusion, disorientation and multisystemic sarcoidosis. (a) Axial T1-weighted MR image at the level of the basal ganglia is unremarkable. (b) Axial T2 and (c) axial FLAIR images show areas of increased signal intensity within the white matter of the medial frontal lobes, left being greater than right. (d) Axial gadolinium-enhanced image shows minimal enhancement in the medial frontal lobes. 


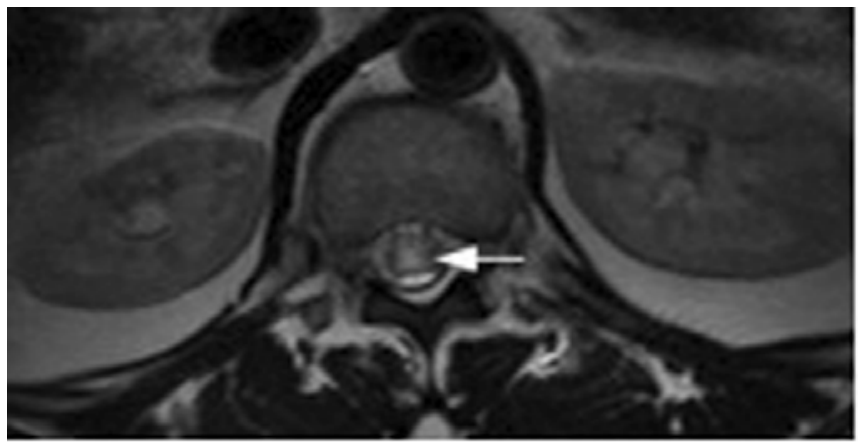

(a)

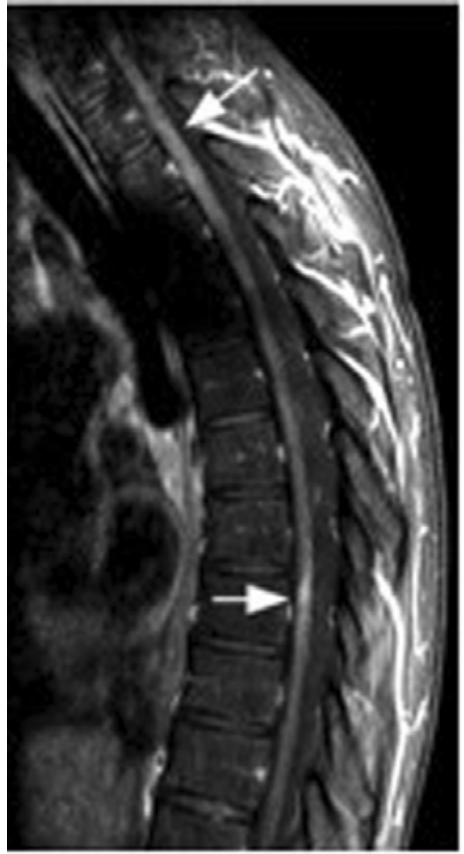

(b)

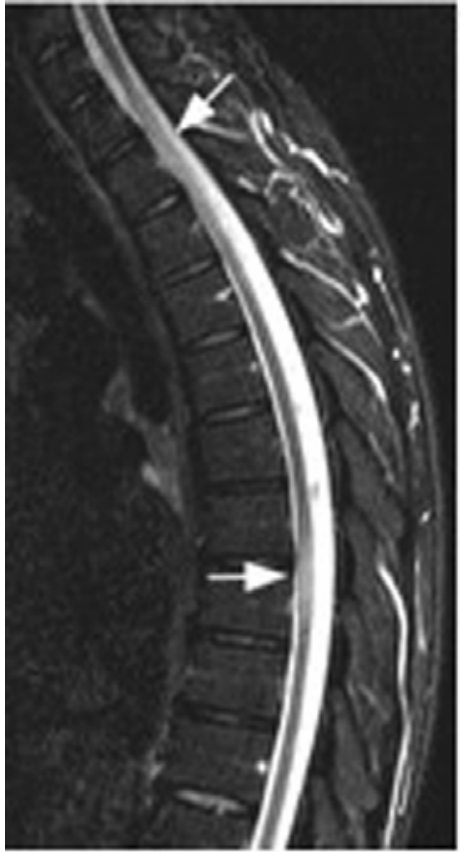

(c)

Fig. 23. Intramedullary sarcoidosis. (a) A 27-year-old male with sarcoidosis. Axial T2weighted image at the level of the conus medullaris shows an area of increased signal intensity within the conus (arrow) representing intramedullary involvement. (b) A 27year-old male with systemic sarcoidosis presented with paraplegia. A T2-weighted sagittal image shows patchy increased signal intensity within the spinal cord at the C7-T2 level as well as T6-T7 level (arrows) and (c) a sagittal gadolinium-enhanced T1-weighted image shows abnormal enhancement of these lesions (arrows), representing intramedullary involvement. 


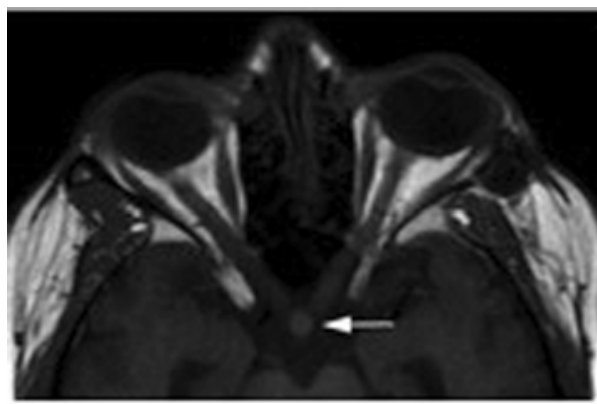

(a)

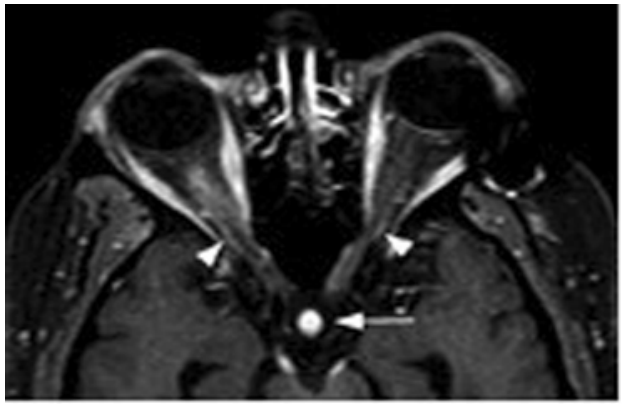

(b)

Fig. 24. A 39-year-old male with vision loss and systemic sarcoidosis. (a) T1-weighted image show s thickened pituitary infundibulum (arrow). (b) Gadolinium-enhanced T1-weighted image shows enhancement of the optic nerves (arrowheads) and pituitary infundibulum (arrow).

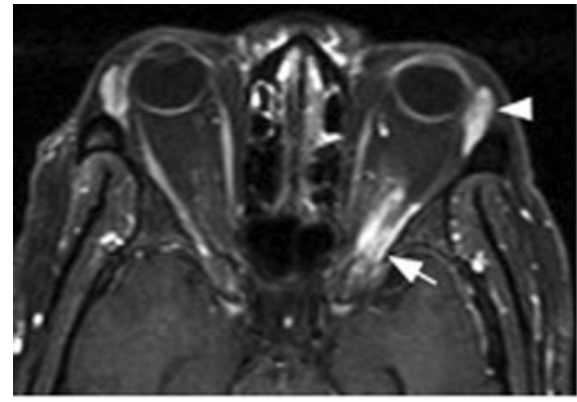

(a)

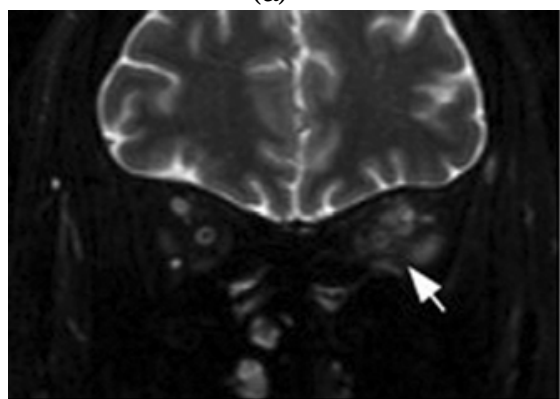

(c)

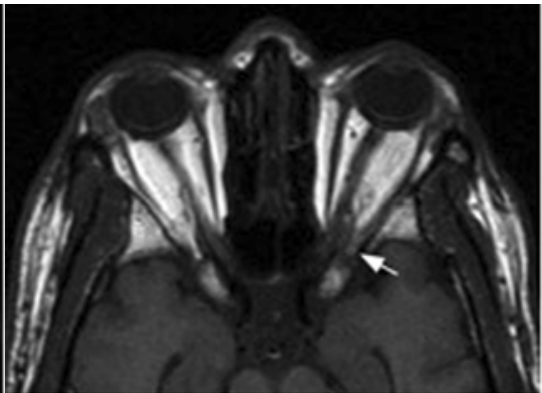

(b)

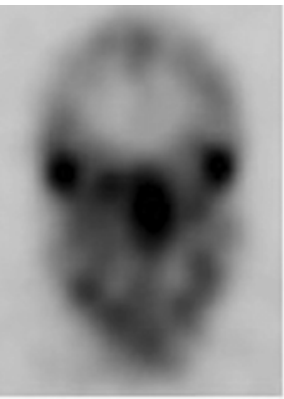

(d)

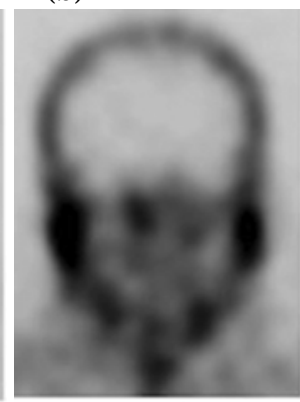

(e)

Fig. 25. A 44-year-old male with pulmonary sarcoidosis, left eye pain and sixth nerve palsy. (a) Axial T1-weighted image shows a low signal intensity lesion in the left orbital apex (arrow). (b) Gadolinium-enhanced fat suppressed T1-weighted image shows enhancement of the previously seen lesion (arrow). Slight enlargement of the left lacrimal gland is noted (arrowhead). (c) Coronal fat suppressed T2-weighted image shows diffuse increased signal intensity with in the left intraconal fat, representing an inflammatory process (arrow). (d) and (e) ${ }^{67}$ Gallium SPECT images show increased radiopharmaceutical activity in the lacrimal and parotid glands respectively. 


\subsection{Dural Involvement}

Diffuse thickening of the dura and focal nodular dural masses are common manifestations of dural sarcoidosis. Dural involvement with sarcoidosis is much more commonly found in the brain rather than in the spine. In the brain, these lesions appear dark on T2-weighted sequences and enhance diffusely on contrast-enhanced T1-weighted sequences (Figure 26). Unlike in leptomeningeal disease, involvement of the basal cisterns and perivascular spaces are not seen. When seen in the spine, dural-based high signal intensity masses on T2weighted sequences are characteristic (Figure 27).

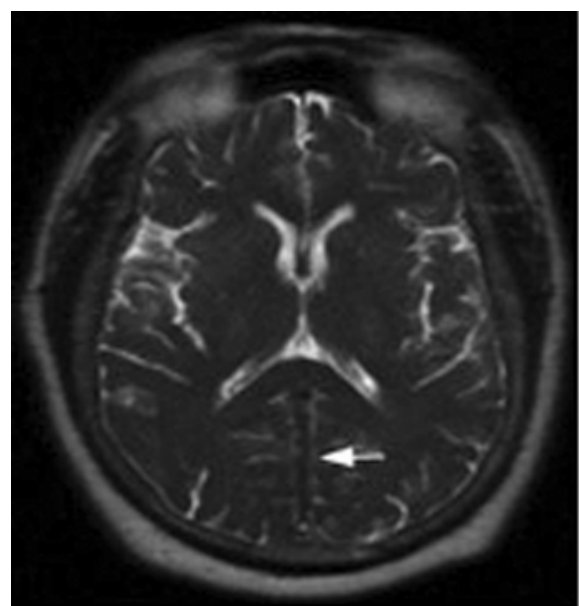

(a)

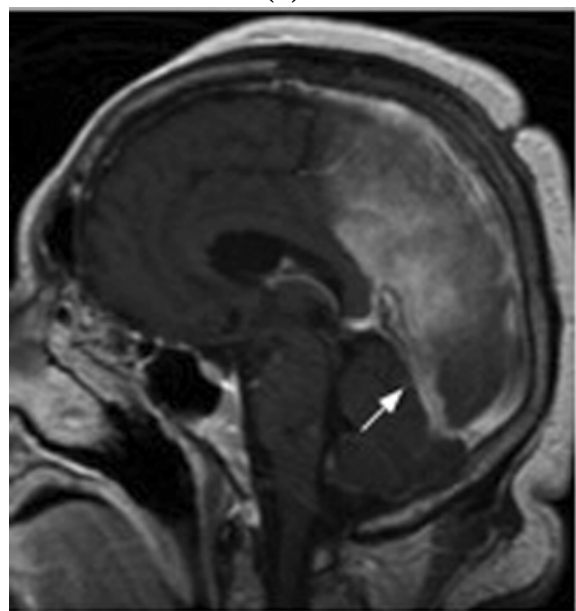

(c)

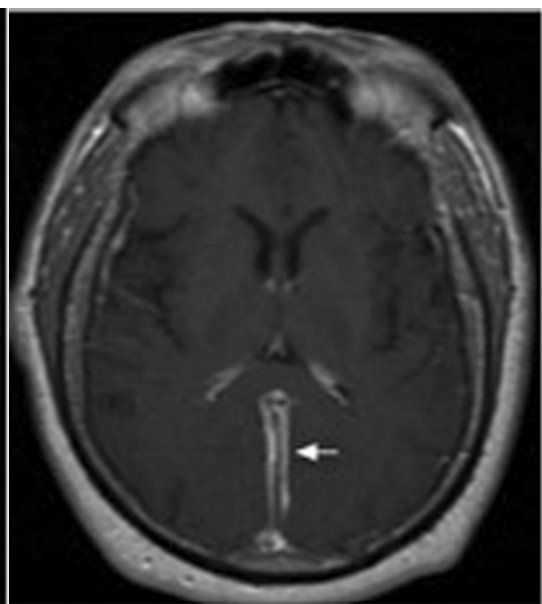

(b)

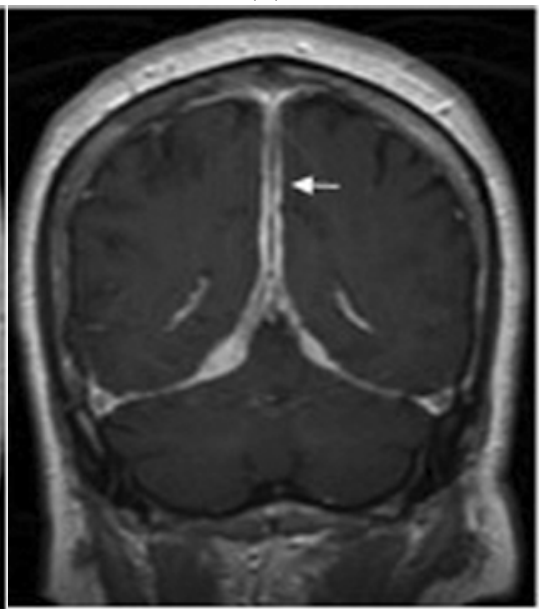

(d)

Fig. 26. A 66-year-old male with worsening headaches and sarcoidosis. (a) A T2-weighted image shows diffuse low signal intensity in the dura. (b), (c), and (d) Axial, sagittal and coronal gadolinium-enhanced images demonstrate diffuse and nodular enhancement of the dura respectively (arrows). 


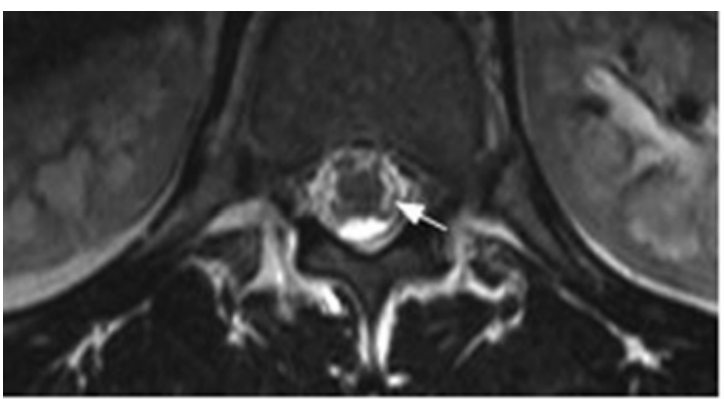

(a)

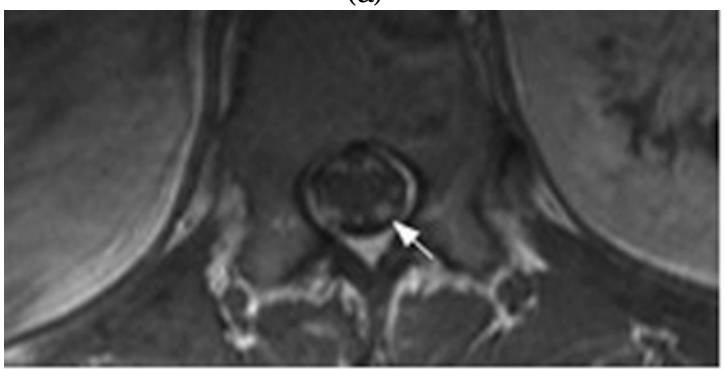

(b)

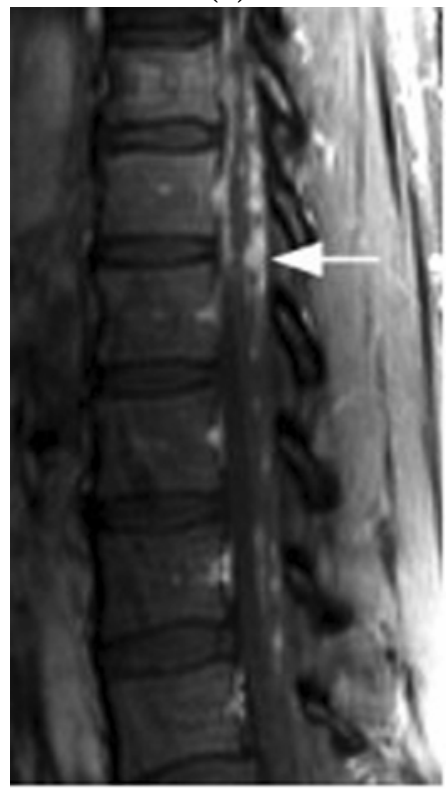

(c)

Fig. 27. A 27-year-old male with confusion and thoracic sarcoidosis. (a) T2-weighted image show s irregular areas of increased signal intensity (arrow) at the surface of the cauda equina. (b) Axial and (c) sagittal gadolinium enhanced T1-weighted images show nodular enhancement (arrows) compatible with dural involvement. 
Refractory and recurrent CNS sarcoidosis is common (Christoforidis et al., 1999). Imaging characteristics of neurosarcoidosis are nonspecific and correlation between a response to treatment and resolution of imaging findings is not well established. Nonetheless, imaging can be useful for follow-up in patients with known CNS sarcoidosis. Lexa et al evaluated the role of MRI in the diagnosis and treatment of 24 patients with neurosarcoidosis. White matter and periventricular high signal intensity on T2-weighted sequences, leptomeningeal enhancement, parenchymal mass, lacrimal gland mass, hydrocephalus, ventriculomegaly, periventricular enhancement, extraaxial mass, optic chiasmal enhancement, nerve root enhancement, enlarged pituitary infundibulum, and pontine infarct were identified. They concluded that enhancement was a useful clue to the diagnosis in 15 of 17 cases in which gadolinium was used (88\%). Additionally, it was shown that MRI is useful in the evaluation of treatment response in patients with neurosarcoidosis particularly in the leptomeningeal and parenchymal lesions (Lexa \& Grossman, 1994).

Symptomatic neurosarcoidosis may be present in $3 \%-5 \%$ of patients with sarcoidosis. Although imaging findings are nonspecific, recognition of common patterns may be helpful in the planning for further diagnostic tests and disease surveillance. MRI with gadolinium is the study of choice for initial diagnosis as well as follow-up evaluation of neurosarcoidosis.

\section{Musculoskeletal sarcoidosis}

Although sarcoidosis can involve any organ, symptomatic musculoskeletal involvement is uncommon. More specifically, muscular, articular and osseous sarcoidosis can be seen. Imaging findings are nonspecific in most cases.

\subsection{Muscular involvement}

Symptomatic myopathy is an extremely rare manifestation of sarcoidosis, which is seen in less than $0.5 \%$ of patients (Douglas et al., 1973). However, asymptomatic granulomatous disease is seen commonly in up to $80 \%$ of patients (Baydur et al., 1993). Chronic myopathy, palpable lesions and acute myositis are different presentations of symptomatic muscular involvement with sarcoidosis. Typically, sarcoid myopathy has a symmetrical pattern with bilateral involvement of the proximal muscles of the extremities. Nevertheless, any muscular structure including the respiratory muscles can be involved.

Oval nodules with a central area of low signal intensity surrounded by an area of increased signal intensity in T2-weighted images are characteristic for nodular sarcoidosis. Evaluation of sonographic and MRI findings in three cases of the nodular type of muscular sarcoidosis revealed intramuscular hypoechoic well-defined nodules in all three patients (Tohme-Noun et al., 2004). On MRI, the nodules were iso- or hyperintense on T1-weighted sequences. On T2-weighted images and STIR sequences, intramuscular nodules of homogeneous high signal intensity were observed. Homogeneous enhancement in all nodules on gadoliniumenhanced T1-weighted sequences was characteristic.

In chronic myopathy, MRI and CT occasionally show muscle atrophy. With acute myositis, on MR imaging diffuse increased T2 signal intensity of involved muscles may be noted. CT may show thickening of the involved musculature (Figure 28 ). ${ }^{67} \mathrm{Ga}$ scintigraphy reveals nonspecific diffuse increased radiopharmaceutical uptake in the involved musculature in both acute myositis and chronic myopathy. 


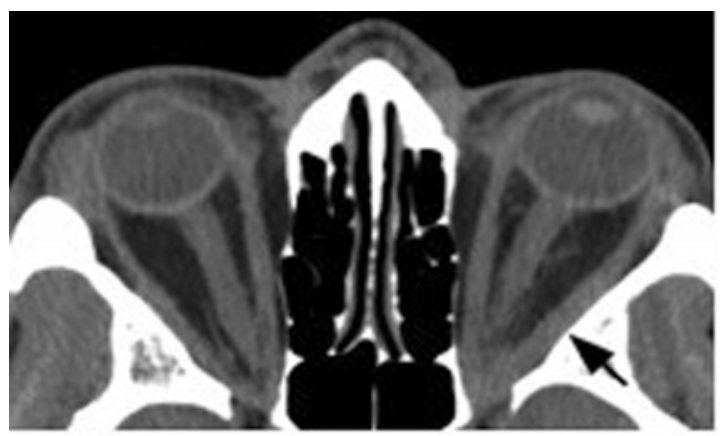

Fig. 28. A 44-year-old male with left eye pain and sarcoidosis. Axial CT image shows mild thickening of the left lateral rectus muscle (arrow) compatible with granulomatous infiltration.

\subsection{Joint involvement}

Arthralgia with polyarticular involvement of the distal joints of the extremities is a common early finding of sarcoidosis. Additionally, MRI findings such as synovitis, bursitis, tenosynovitis, and tendonitis have been reported in patients with sarcoidosis (Figure 29). Tissue biopsy might be necessary for definite diagnosis as these findings are nonspecific.

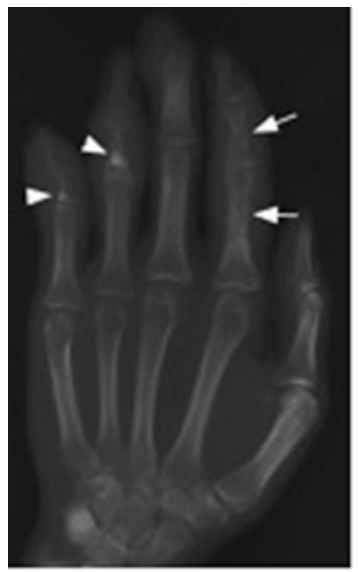

Fig. 29. Posteroanterior radiograph of the right hand shows sausage-like dactylitis of the second to fifth digits in a patient with sarcoidosis, representing synovitis. Multiple classic osteolytic bony lesions (arrows) as well as sclerotic foci at the base of the middle phalanges of the forth and fifth digits (arrowheads) are also noted. (Courtesy of Drs. Shipley, Weinstein and Wissman. University of Cincinnati, Ohio, USA)

\subsection{Osseous involvement}

Osseous sarcoidosis has been reported in about $5 \%$ of patients (Johns \& Michele, 1999). Plain radiographs are the first imaging modality for diagnosis of osseous sarcoidosis. Osteolysis with a lacy trabecular pattern is the characteristic findings on radiographs (Figure 30). 
Typically, small bones of the hand and foot are involved although the vertebral body (Figure 31), skull, and long bones may also be affected rarely. Cyst formation with a "punched out" pattern appearing as well-circumscribed lytic lesions with nonsclerotic margins is also common. Pathologic fractures secondary to extensive bony erosion have been reported (Figure 32). Periosteal reaction is usually not present. Furthermore, osseous sarcoidosis can also present as osteosclerosis, as evidenced by nodular opacities in the medullary cavity on plain radiographs (Figure 29).

99m Tc-pyrophosphate scintigraphy is more sensitive in detecting osseous sarcoidosis in comparison to plain radiographs. Areas of increased radiopharmaceutical activity correspond to active granulomatous infiltration. Nuclear imaging findings are nonspecific for sarcoidosis; however ${ }^{99 \mathrm{~m} T c}$ bone scintigraphy can be used effectively for monitoring disease activity.

MRI is the most sensitive imaging modality for detecting osseous sarcoidosis. Typically, sarcoid bony lesions appear hypointense on T1-weighted sequences and hyperintense on T2-weighted, inversion-recovery, and fat-saturated proton-density-weighted MR sequences. Occasionally, the lesions can have low signal intensity.

Occult lesions, soft tissue involvement and cortical disruption may also be identified with MR imaging in osseous sarcoidosis. In the long bones, sarcoidosis can be seen as welldefined discrete masses, infiltrative marrow lesions and ill-defined mass-like lesions, which may enhance after administration of contrast. These lesions can be replaced with fat or fibrotic tissues, indicating the chronicity of the disease.

In vertebral bodies lytic lesions with sclerotic margins are typical which may extend into the pedicles. Sclerotic or mixed lytic and sclerotic involvement as well as disc involvement is all relatively rare. In the skull, sarcoid lesions are usually well-defined expansile lytic areas with a "punched out" pattern, which can be visualized on CT. Skull lesions are seldom seen alone and are customarily associated with other bony lesions when osseous involvement of sarcoidosis is present (Yaghmai, 1983).

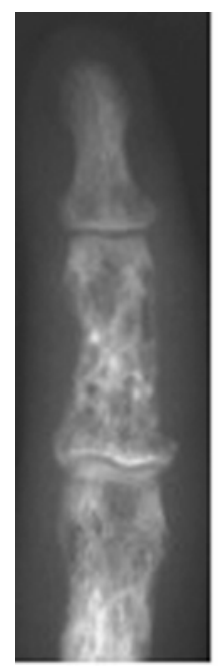

Fig. 30. Coned-down posteroanterior radiograph of the right hand in a patient with sarcoidosis shows osteolytic lesions with a lacy pattern within the middle phalanx of the second digit. (Courtesy of Drs. Shipley, Weinstein and Wissman, University of Cincinnati, Cincinnati, Ohio, USA) 

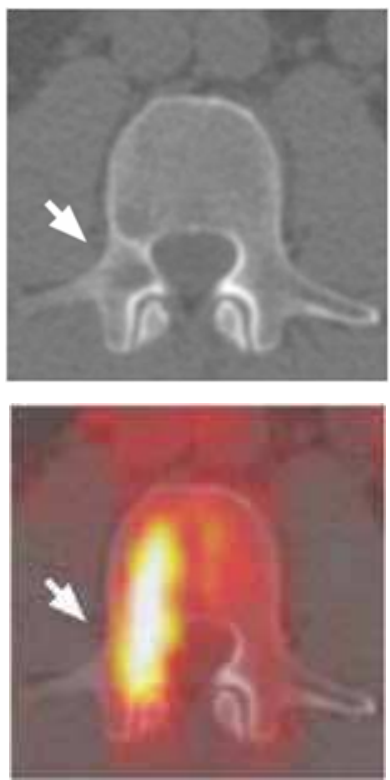

Fig. 31. A 50-year-old male with sarcoiodosis. PET/ CT images show a lytic lesion with increased FDG activity within the posterior aspect of the L3 vertebral body. (Rajebi et al. Uncommon osseous involvement in multisystemic sarcoidosis. Ann Saudi Med. 2009;29:485-6)

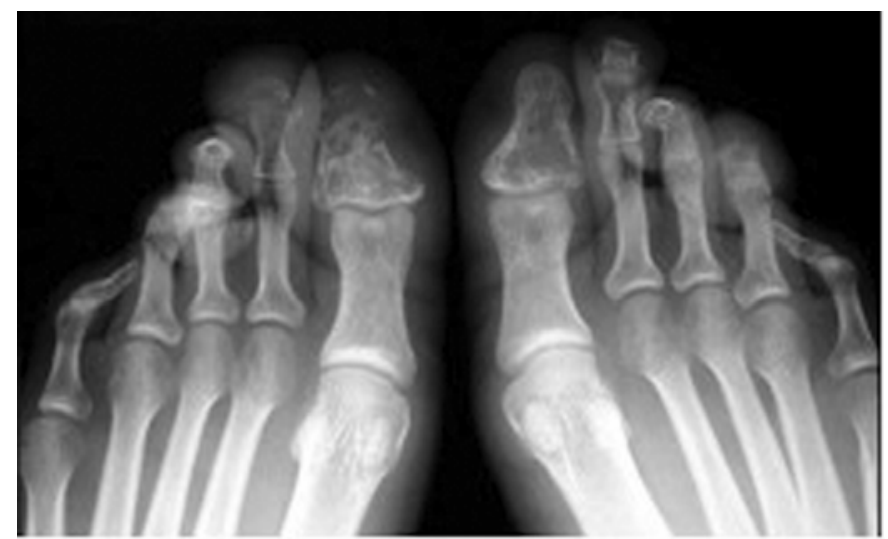

Fig. 32. Coned-down posteroanterior radiograph of the feet in a patient with sarcoidosis shows a large osteolytic lesion in the distal phalanx of the right first digit causing a pathological fracture. (Courtesy of Dr. Hojnow ski, SUNY Upstate Medical University, Syracuse, New York, USA)

Compared to conventional radiography and nuclear scintigraphy, MRI with gadolinium is more sensitive in detection of lesions in patients with musculoskeletal sarcoidosis. Nonetheless, in most cases these findings are nonspecific and correlation with clinical and laboratory findings is essential. 


\section{FDG-PET and PET/CT}

FDG-PET and PET/ CT identify areas of abnormally increased glucose transport and metabolism. This capability makes FDG-PET imaging a suitable modality in conditions with increased glucose metabolism such as cancer and inflammatory disease. However, pattern and intensity of FDG uptake are nonspecific in multisystemic disorders such as sarcoidosis and can be mistaken with lymphoma and diffuse metastatic diseases (Figure 31 and 33) (Rajebi et al., 2009). Despite this, FDG uptake value can decrease after treatment of sarcoidosis and FDG-PET and PET/ CT can be useful for monitoring the disease and efficacy of the treatment (Brundin et al., 1994).

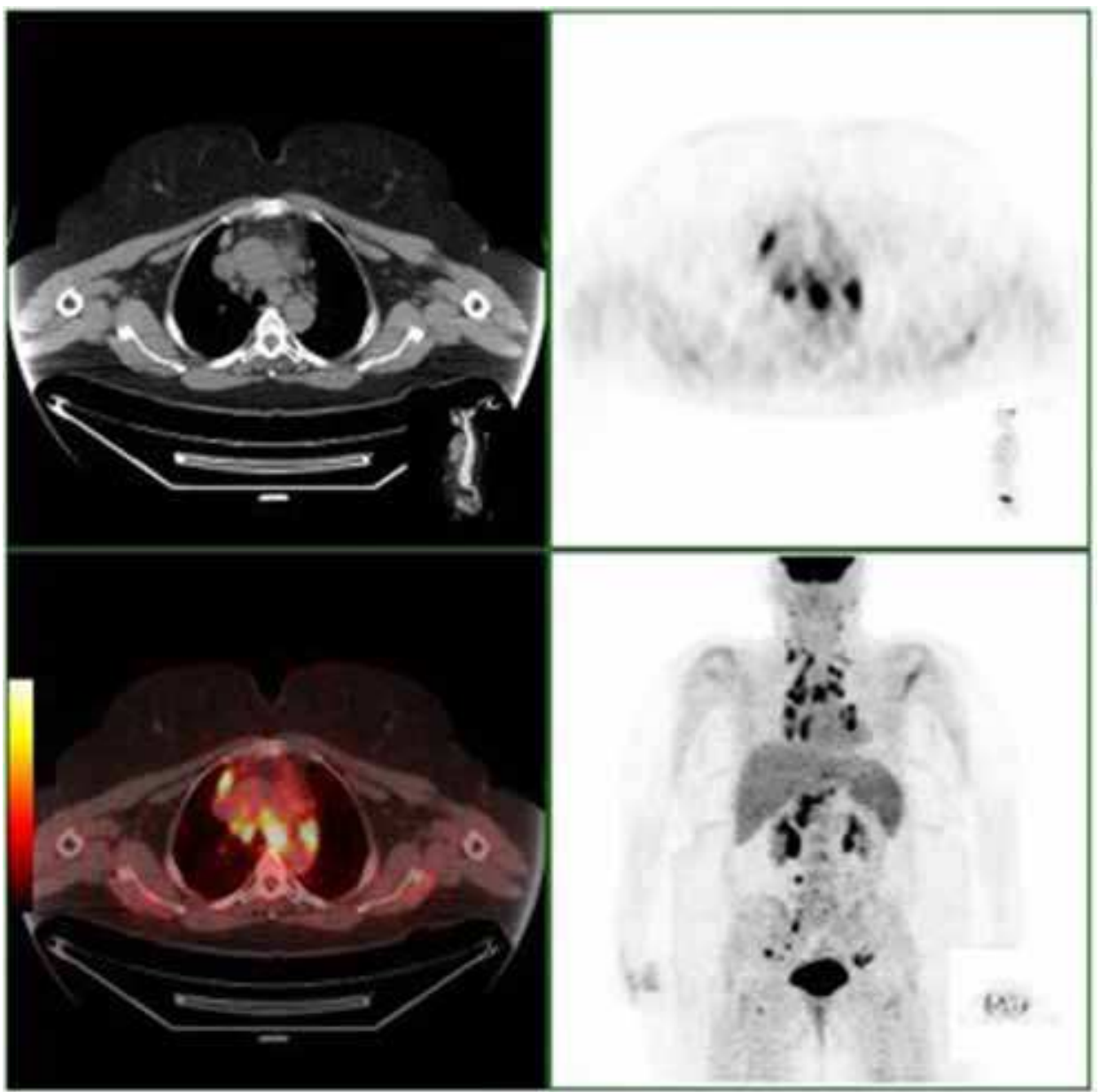

Fig. 33. A 37-year-old female with mediastinal lymphadenopathy on chest radiograph (not shown). An FDG-PET/ CT shows extensive FDG-avid hilar, mediastinal, intrabdominal and inguinal lymphadenopathy. Biopsy confirmed the diagnosis of sarcoidosis. (Courtesy of Dr. McGrath, SUNY Upstate Medical University, Syracuse, New York, USA) 
It has been shown that persistent retention of FDG correlates with refractory disease. Umeda et al evaluated the prognostic value of dual time point FDG-PET in patients with pulmonary sarcoidosis. In this study, twenty-one patients with pulmonary sarcoidosis underwent an early FDG-PET at 60 minutes and a delayed FDG-PET scan at 180 minutes after the injection of FDG. Standardized uptake values (SUVs) at the two time points and the retention index (RI-SUV) calculated from these were assessed. To evaluate disease progression, all patients underwent chest CT one year after the initial FDG-PET. RI-SUV was significantly higher in patients with increased or unchanged pulmonary lesions at follow-up CT than in patients with improved pulmonary lesions indicating that RI-SUV can be a good indicator of disease progression (Umeda et al 2011).

With FDG-PET/ CT, it is postulated that the addition of typical CT findings in patients with multisystemic disease such as hilar and paratracheal lymphadenopathy and parenchymal involvement in pulmonary sarcoidosis, can increase usefulness of this modality in evaluation of treatment response in sarcoidosis.

\section{Conclusion}

Sarcoidosis is a multisystemic disease, which can involve any organ in the body. Similar to presenting signs and symptoms, imaging findings of sarcoidosis are diverse. Conventional radiography is useful in initial evaluation of pulmonary and musculoskeletal sarcoidosis. Ultrasonography is playing an emerging role in the diagnosis of pulmonary involvement. The modality of choice for evaluation of the parenchymal lung disease is CT. MRI findings in cardiac sarcoidosis are relatively specific and advantageous in diagnosis. Furthermore, MRI is an extremely invaluable tool for initial diagnosis and treatment response in neurosarcoidosis. Extent of musculoskeletal sarcoidosis is also best evaluated with MRI. Although the imaging findings in abdominal sarcoidosis are nonspecific, CT and ultrasonography are helpful in evaluation of organ involvement and lymphadenopathy. FDG-PET/ CT may be useful in evaluating disease progress and treatment efficacy.

Clinicians and radiologists should be familiar with typical as well as atypical imaging findings of sarcoidosis. This may decrease the unnecessary workup and prevent needless patient discomfort.

\section{References}

Abehsera M, Valeyre D, Grenier P, Jaillet H, Battesti JP, \& Brauner MW. (2000). Sarcoidosis with pulmonary fibrosis: CT patterns and correlation with pulmonary function. A.R Am JRoentgenol, Vol. 174, No. 6, (June 2000), pp. 1751-7, ISSN 0361-803X

Baydur A, Pandya K, Sharma OP, Kanel GC, \& Carlson M. (1993). Control of ventilation, respiratory muscle strength, and granulomatous involvement of skeletal muscle in patients with sarcoidosis. Chest, Vol. 103, No. 2, (February 1993), pp. 396-402, ISSN 0012-3692

Boeck, C. (1899). Multiple benign sarkoid of the skin. I Cutan. Genitourinary Dis, Vol. 17, pp. $543-550$ 
Britt AR, Francis IR, Glazer GM, \& Ellis JH.(1991). Sarcoidosis: abdominal manifestations at CT. Radiology, Vol.178, No. 1, (January 1991), pp. 91-94, ISSN 0033-8419

Brudin LH, Valind SO, Rhodes CG, Pantin CF, Sweatman M, Jones T, \& Hughes $\mathbb{M}$. (1994). Fluorine-18 deoxyglucose uptake in sarcoidosis measured with positron emission tomography. Eur JNucl Med, Vol. 21, No. 4, (April 1994), pp. 297-305, ISSN 03406997

Carmody RF, Mafee MF, Goodwin JA, Small K, \& Haery C. (1994). Orbital and optic pathway sarcoidosis: MR findings. A.NR Am JNeuroradiol, Vol. 15, No. 4, (April 1994), pp. 775-83, ISSN 0195-6108

Christoforidis GA, Spickler EM, Reccio MV, \& Mehta BM. (1999). MR of CNS sarcoidosis: correlation of imaging features to clinical symptoms and response to treatment. A.NR Am JNeuroradiol, Vol. 20, No. 4, (April 1999), pp. 655-669, ISSN 0195-6108

Cohen-Aubart F, Galanaud D, Grabli D, Haroche J, Amoura Z, Chapelon-Abric C, LyonCaen O, Valeyre D, \& Piette JC.(2010). Spinal cord sarcoidosis: clinical and laboratory profile and outcome of 31 patients in a case-control study. Medicine (Baltimore), Vol. 89, No. 2, (March 2010), pp. 133-40, ISSN 0025-7974

Criado E, Sánchez M, Ramírez J, Arguis P, de Caralt TM, Perea RJ, \& Xaubet A.(2010). Pulmonary sarcoidosis: typical and atypical manifestations at high-resolution CT with pathologic correlation. Radiographics, Vol. 30, No. 6, (October 2010), pp.156786, ISSN 1527-1323

Douglas AC, Macleod JG, \& Matthews JD. (1973). Symptomatic sarcoidosis of skeletal muscle. JNeurol Neurosurg Psychiatry, Vol. 36, No. 6, (December 1973), pp. 1034-40, ISSN 0022-3050

Garwood S, Judson MA, Silvestri G, Hoda R, Fraig M, \& Doelken P. (2007). Endobronchial ultrasound for the diagnosis of pulmonary sarcoidosis. Chest, Vol. 132, No. 4, (October 2007), pp. 1298-304, ISSN 0012-3692

Handa T, Nagai S, Fushimi Y, et al. (2006). Clinical and radiographic indices associated with airflow limitation in patients with sarcoidosis. Chest, Vol. 130, No. 6, (December 2006), pp. 1851-1856, ISSN 0012-3692

Hansell DM, Bankier AA, MacMahon H, McLoud TC, Muller NL, \& Remy J. (2008). Fleischner Society: glossary of terms for thoracic imaging. Radiology, Vol. 246, No. 3, (March 2008), pp. 697-722, ISSN 1527-1315

Hutchinson, J. (1877). Case of livid papillary psoriasis. In Illustrations of Clinical Surgery, Vol. 1, pp. 42-43, J\&A Churchill, London, UK

Ichinose A, Otani H, Oikawa M, Takase K, Saito H, Shimokawa H, \& Takahashi S. (2008). MRI of cardiac sarcoidosis: basal and subepicardial localization of myocardial lesions and their effect on left ventricular function. AeR Am JRoentgenol, Vol. 191, No. 3, (September 2008), pp.862-9, ISSN 1546-3141

Iwai K, Tachibana T, Hosoda Y, \& Matsui Y. (1988). Sarcoidosis autopsies in Japan: frequency and trend in the last 28 years. Sarcoidosis, Vol. 5, No. 1, (March 1988), pp. 60-65, ISSN 0393-1447

Johns CJ \& Michele TM. (1999). The clinical management of sarcoidosis. A 50-year experience at the Johns Hopkins Hospital. Medicine (Baltimore), Vol. 78, No. 2, (March 1999), pp. 65-111, ISSN 0025-7974 
Junger SS, Stern BJ, Levine SR, Sipos E, \& Marti-Masso JF. (1993). Intramedullary spinal sarcoidosis: clinical and magnetic resonance imaging characteristics. Neurology, Vol. 43, No. 2, (February 1993), pp. 333-337, ISSN 0028-3878

Kuznitsky, E. \& A. Bittorf. (1915). Sarkoid mit Beteiligung innerer Organe. Munch. Med. Wochenschr, pp.1349-1353

Lexa FJ \& Grossman RI. (1994). MR of sarcoidosis in the head and spine: spectrum of manifestations and radiographic response to steroid therapy. A.NR Am J Neuroradiol, Vol. 15, No. 5, (May 1994), pp. 973-82, ISSN 0195-6108

Lynch $\mathbb{P}$ 3rd, Kazerooni EA, \& Gay SE. (1997). Pulmonary sarcoidosis. Clin Chest Med, Vol. 18, No. 4, (December 1997), pp. 755-785, ISSN 0272-5231

Nowak DA \& Widenka DC. (2001). Neurosarcoidosis: a review of its intracranial manifestation. JNeurol, Vol. 248, No. 5, (May 2001), pp. 363-372, ISSN 0340-5354

Okumura W, Iwasaki T, Toyama T, Iso T, Arai M, Oriuchi N, Endo K, Yokoyama T, Suzuki T, \& Kurabayashi M. (2004). Usefulness of fasting 18F-FDG PET in identification of cardiac sarcoidosis. JNucl Med, Vol. 45, No. 12, (December 2004), pp. 1989-98, ISSN 0161-5505

Perry A \& Vuitch F. (1995). Causes of death in patients with sarcoidosis: a morphologic study of 38 autopsies with clinicopathologic correlations. Arch Pathol Lab Med, Vol. 119, No. 2, (February 1995), pp. 167-172, ISSN 0003-9985

Rajebi MR, Shahrokni A, \& Chaisson M. (2009). Uncommon osseous involvement in multisystemic sarcoidosis. Ann Saudi Med, Vol 29, No. 6, (Nov-Dec 2009), pp. 485-6, ISSN 0975-4466

Siltzbach LE. (1967). Sarcoidosis: clinical features and management. Med Clin North Am, Vol. 51, No. 2, (March 1967), pp. 483-502, ISSN 0025-7125

Soskel NT \& Sharma OP. (2000). Pleural involvement in sarcoidosis. Curr Opin Pulm Med, Vol. 6, No. 5, (September 2000), pp. 455-468, ISSN 1070-5287

Sulavik SB, Spencer RP, Weed DA, Shapiro HR, Shiue ST, \& Castriotta RJ.(1990). Recognition of distinctive patterns of gallium-67 distribution in sarcoidosis. JNucl Med, Vol. 31, No. 12 (December 1990), pp. 1909-14, ISSN 0161-5505

Tohme-Noun C, Le Breton C, Sobotka A, Boumenir ZE, Milleron B, Carette MF, \& Khalil A. (2004). Imaging findings in three cases of the nodular type of muscular sarcoidosis. A.R Am JRoentgenol, Vol. 183, No. 4, (October 2004) pp. 995-9, ISSN 0361-803X

Terasaki H, Fujimoto K, Müller NL, Sadohara J, Uchida M, Koga T, Aizawa H, \& Hayabuchi N. (2005). Pulmonary sarcoidosis: comparison of findings of inspiratory and expiratory high-resolution CT and pulmonary function tests between smokers and nonsmokers. A.R Am JRoentgenol, Vol. 185, No. 2, (August 2005), pp. 333-8, ISSN 0361-803X

Umeda Y, Demura Y, Morikawa M, Ameshima S, Tsuchida T, Fujibayashi Y, Okazawa H, Ishizaki T. (2011) Prognostic value of dual time point (18) F-fluorodeoxyglucose positron emission tomography in patients with pulmonary sarcoidosis. Respirology, "Accepted Article"; doi: 10.1111/ j.1440-1843.2011.01966.x

Warshauer DM \& Lee $\mathbb{J K}$. (2004). Imaging manifestation of abdominal sarcoidosis. AdR Am $J$ Roentgenol, Vol. 182, No. 1, (January 2004), pp. 15-28, ISSN 0361-803X 
Wurm, K, H. Reindell, \& L. Heilmeyer. (1958). Der Lungenboeck im Röntgenbild. Thieme, Stuttgart, Germany.

Vignaux O. (2005). Cardiac sarcoidosis: spectrum of MRI features. AdR Am JRoentgenol, Vol. 184, No. 1, (January 2005), pp. 249-54, ISSN 0361-803X

Yaghmai I.(1983). Radiographic, angiographic and radionuclide manifestations of osseous sarcoidosis. Radiographic, Vol. 3, No. 3, (September 1983), pp. 375-396, ISSN 15271323 


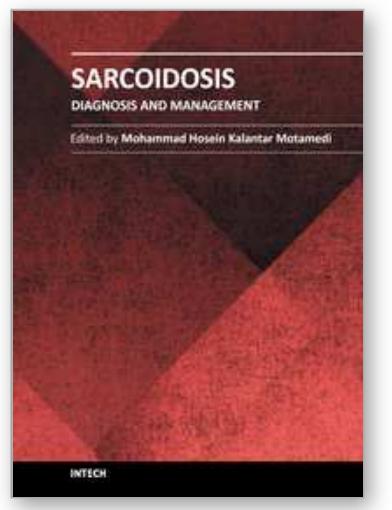

\author{
Sarcoidosis Diagnosis and Management \\ Edited by Prof. Mohammad Hosein Kalantar Motamedi
}

ISBN 978-953-307-414-6

Hard cover, 280 pages

Publisher InTech

Published online 17, October, 2011

Published in print edition October, 2011

Sarcoidosis is a type of inflammation that occurs in various locations of the body for no known reason. Normally, when foreign substances or organisms enter the body, the immune system will fight back by activating an immune response. Inflammation is a normal part of this immune response, but it should subside once the foreign antigen is gone. In sarcoidosis, the inflammation persists, and some of the immune cells form abnormal clumps of tissue called granulomas. The disease can affect any organ in the body, but it is most likely to occur in the lungs. It can also affect the skin, eyes, liver, or lymph nodes. Although the cause of sarcoidosis is not known, research suggests that it may be due to an extreme immune response or extreme sensitivity to certain substances. It also seems to have a genetic component as well, and tends to run in families. Sarcoidosis most commonly develops in people between 20 and 50 years of age. African Americans are somewhat more likely to develop sarcoidosis than Caucasians, and females are somewhat more likely to develop sarcoidosis than males. The symptoms of sarcoidosis depend on the organ involved. This book deals with the diagnosis and treatment of this mysterious disease of unknown etiology.

\title{
How to reference
}

In order to correctly reference this scholarly work, feel free to copy and paste the following:

M. Reza Rajebi, Nicole A. Zimmerman, Roozbeh Sharif, Ernest M. Scalzetti, Stuart A. Groskin and Rolf A. Grage (2011). Imaging in Sarcoidosis, Sarcoidosis Diagnosis and Management, Prof. Mohammad Hosein Kalantar Motamedi (Ed.), ISBN: 978-953-307-414-6, InTech, Available from:

http://www.intechopen.com/books/sarcoidosis-diagnosis-and-management/imaging-in-sarcoidosis

\section{INTECH}

open science | open minds

\section{InTech Europe}

University Campus STeP Ri

Slavka Krautzeka 83/A

51000 Rijeka, Croatia

Phone: +385 (51) 770447

Fax: +385 (51) 686166

www.intechopen.com

\section{InTech China}

Unit 405, Office Block, Hotel Equatorial Shanghai

No.65, Yan An Road (West), Shanghai, 200040, China 中国上海市延安西路65号上海国际贵都大饭店办公楼405单元

Phone: +86-21-62489820

Fax: $+86-21-62489821$ 
(C) 2011 The Author(s). Licensee IntechOpen. This is an open access article distributed under the terms of the Creative Commons Attribution 3.0 License, which permits unrestricted use, distribution, and reproduction in any medium, provided the original work is properly cited. 